\title{
Unified approach to catastrophic events: from the normal state to geological or biological shock in terms of spectral fractal and nonlinear analysis
}

\author{
K. A. Eftaxias ${ }^{1}$, P. G. Kapiris ${ }^{1}$, G. T. Balasis ${ }^{2}$, A. Peratzakis ${ }^{1}$, K. Karamanos $^{3}$, J. Kopanas ${ }^{1}$, G. Antonopoulos ${ }^{1}$, and \\ K. D. Nomicos ${ }^{4}$ \\ ${ }^{1}$ Solid State Section, Physics Department, University of Athens, Panepistimiopolis, 157-84, Zografos, Athens, Greece \\ ${ }^{2}$ Department of Earth's Magnetic Field, GeoForschungsZentrum Potsdam, Telegrafenberg, 14473, Potsdam, Germany \\ ${ }^{3}$ Centre for Nonlinear Phenomena and Complex Systems, Université Libre de Bruxelles, Campus Plaine, C.P. 231, Boulevard \\ du Triomphe, B-1050, Brussels, Belgium \\ ${ }^{4}$ Department of Electronics, Technological Education Institute of Athens, Egaleo, 12210, Greece
}

Received: 3 August 2005 - Revised: 27 January 2006 - Accepted: 27 January 2006 - Published: 3 April 2006

Part of Special Issue "Seismic hazard evaluation, precursory phenomena and seismo electromagnetics"

\begin{abstract}
An important question in geophysics is whether earthquakes (EQs) can be anticipated prior to their occurrence. Pre-seismic electromagnetic (EM) emissions provide a promising window through which the dynamics of EQ preparation can be investigated. However, the existence of precursory features in pre-seismic EM emissions is still debatable: in principle, it is difficult to prove associations between events separated in time, such as EQs and their EM precursors. The scope of this paper is the investigation of the pre-seismic EM activity in terms of complexity. A basic reason for our interest in complexity is the striking similarity in behavior close to irreversible phase transitions among systems that are otherwise quite different in nature. Interestingly, theoretical studies (Hopfield, 1994; Herz and Hopfield 1995; Rundle et al., 1995; Corral et al., 1997) suggest that the EQ dynamics at the final stage and neural seizure dynamics should have many similar features and can be analyzed within similar mathematical frameworks. Motivated by this hypothesis, we evaluate the capability of linear and non-linear techniques to extract common features from brain electrical activities and pre-seismic EM emissions predictive of epileptic seizures and EQs respectively. The results suggest that a unified theory may exist for the ways in which firing neurons and opening cracks organize themselves to produce a large crisis, while the preparation of an epileptic shock or a large EQ can be studied in terms of "Intermittent Criticality".
\end{abstract}

Correspondence to: K. Eftaxias

(ceftax@phys.uoa.gr)

\section{Introduction}

Fracture in disordered media is a complex problem which still lacks a definite physical and theoretical treatment. Despite the large amount of experimental data and the considerable effort that has been undertaken by the material scientists, many questions about the fracture remain. Earthquakes (EQs) are large scale fracture phenomena in the Earth's heterogeneous crust. A vital problem in material science and in geophysics is to identify precursors of macroscopic defects or shocks. In physics, the degree to which we can predict a phenomenon is often measured by how well we understand this. Moreover, it is difficult to prove associations between events separated in time, such as EQs and their precursors. Herein, we hope to uncover more information hidden in the pre-seismic (EM) time series, and thus, to achieve a deeper understanding of the physics of the EQ nucleation process.

Both acoustic as well as EM emissions, in a wide frequency spectrum ranging from very low frequencies (VLF) to very high frequencies (VHF), are produced by opening cracks, which can be considered as the so-called precursors of general fracture. We focus on the geophysical view of this problem. Our main tool is the monitoring of the microfractures, which occur before the final break-up in the focal area, by recording their VLF-VHF EM emissions.

In the last 15 years, the study of complex systems has emerged as a recognized field in its own right, although, a good definition of what a complex system has proven elusive. When one considers a phenomenon or a thing that is "complex", one generally associates it with something that is "hard to separate, analyze or to solve". Instead, we refer to

Published by Copernicus GmbH on behalf of the European Geosciences Union. 
"a complex system" as one whose phenomenological laws, which describe the global behavior of the system, are not necessarily directly related to the "microscopic" laws that regulate the evolution of its elementary parts. In other words, "complexity" is the emergence of a non-trivial behavior due to the interactions of the subunits that form the system itself. The statistical features of complex systems are generally not dependent on the details of the interactions of the subunits that form the system. Another relevant ingredient of a complex system is that topological disorder within the system will generally introduce new, surprising effects, different than those one would expect from the simple "microscopic" evolution rules.

A basic reason for our interest in complexity is the striking similarity in behavior close to irreversible phase transitions among systems that are otherwise quite different in nature (Stanley, 1999, 2000; Sornette, 2002; Vicsek, 2001, 2002). One universal footprint seen in many complex systems is self-affinity. The fractional power-law relationship is a classic definition of a self-affine structure. Thus, much work on complexity focuses on statistical power laws that describe the scaling properties of fractal processes and structures. Recent studies have demonstrated that a large variety of complex processes, including EQs (Bak, 1997), forest fires (Malamud et al., 1998), heartbeats (Peng et al., 1995), human coordination (Gilden et al., 1995; Chen et al., 1997), neuronal dynamics (Worrell et al., 2002), financial markets (Mantegna and Stanley, 1995; Lux and Marchesi, 1999), exhibit statistical similarities, most commonly power-law scaling behavior of a particular observable.

A few years ago, Bak et al. $(1987,1989)$ coined the term self-organized criticality (SOC) to describe the phenomenon observed in a particular automaton model, nowadays known as the sandpile model. This system is critical in analogy with classical equilibrium critical phenomena, where neither characteristic time nor length scales exist. The strong analogies between the dynamics of the SOC model for EQs and that of neurobiology have been realized by numerous authors (Hopfield, 1994; Herz and Hopfield, 1995; Corral et al., 1997; Usher et al., 1995; Zhao and Chen, 2002; Plenz, 2003). In general, authors have suggested that EQ dynamics and neurodynamics can be analyzed within similar mathematical frameworks (Herz and Hopfield, 1995; Rundle et al., 2002). Characteristically, driven systems of interconnected blocks with stick-slip friction capture the main features of EQ process. These models, in addition to simulating the aspects of EQs and frictional sliding, can also represent the dynamics of neurological networks (Herz and Hopfield, 1995, and references therein). Hopfield (1994) proposed a model for a network of $N$ integrate-and-fire neurons. In this model, the dynamical equation of $k$ th neuron Eq. (28) in Hopfield (1994) is based on the Hodgekin-Huxley model for neurodynamics and represents the same kind of mean field limit that has been examined in Rundle et al. (2002) in connection with EQs.
We have been motivated by the suggestion that EQ dynamics and neurodynamics can be analyzed within similar mathematical frameworks. We concentrate here on the question whether common precursory symptoms emerge as epileptic seizures and EQs approach.

Electroencephalogram (EEG) time series provide a window through which the dynamics of epileptic seizure preparation can be investigated under well-controlled conditions. Consequently, the analysis of a pure pre-epileptic time series would help in establishing a reliable collection of criteria to indicate the approach to the biological shock. In terms of complexity, such a collection of criteria indicates the approach to global instability in the pre-focal area as well.

The results of the present analysis imply that a unified theory may exist for the ways in which firing neurons/opening cracks organize themselves to produce a significant epileptic seizure/EQ: we show that many similar distinctive symptoms (including common alteration in associated scaling parameters) emerge as epileptic seizures and EQs are approaching. The present state of research requires a refined definition of a possible pre-seismic EM anomaly, as well as the development of more objective methods of distinguishing seismogenic emissions from non-seismic EM events. The observed similarities support the consideration that the detected EM anomaly is originated during the micro-fracturing in the prefocal area of the impending $E Q$.

\section{Pre-seismic electromagnetic activity}

When a heterogeneous material is strained, its evolution towards breaking is characterized by local nucleation and coalescence of micro-cracks. Crack propagation is the basic mechanism of material's failure. The motion of a crack in dynamics fracture has been shown to be governed by a dynamical instability causing oscillations in its velocity and structure on the fracture surface. Experimental evidence indicates that the instability mechanism is that of local branching (Sharon and Fineberg, 1996). A multi-crack state is formed by repetitive, frustrated micro-fracturing events (Sharon and Fineberg, 1999).

In many materials, emission of photons, electrons, ions and neutral particles are observed during the formation of new surface features in fracturing, deformation, wearing, peeling, and so on. Collectively, we refer to these emissions as fractoemission (Langford et al., 1987; Dickinson et al., 1988; Gonzalez and Pantano, 1990; Miura and Nakayama, 2000; Takeuchi and Nagahama, 2004; Mavromatou et al., 2004; Wu et al., 2004). It is worth mentioning that laboratory experiments show that more intense fractoemissions are observed during the unstable crack growth (Gonzalez and Pantano, 1990). The rupture of inter-atomic (ionic) bonds also leads to intense charge separation, the origin of the electric charge between the micro-crack faces. On the faces of a newly created micro-crack the electric charges constitute 

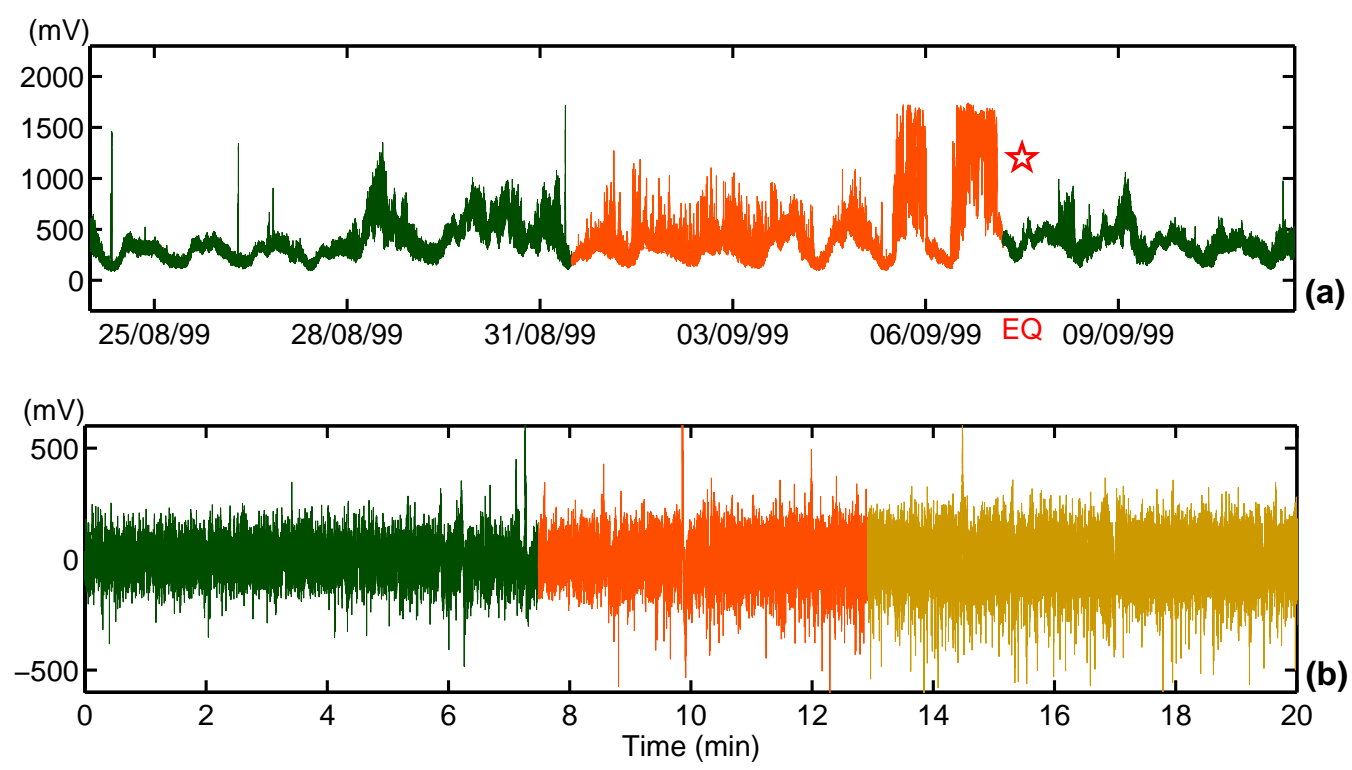

Fig. 1. (a) $10 \mathrm{kHz}$ magnetic field variations associated with the Athens EQ. The consecutive green, red, and green epochs show the normal state, the pre-seismic phase, and the aftershock state (new normal state), correspondingly. The sampling rate was $1 \mathrm{~Hz}$. (b) Rat EEG time series. The sampling rate was $200 \mathrm{~Hz}$. Bicuculline i.p. injection was used to induce the rat epileptic seizures. The green, red and ochre epochs show the normal state, the pre-epileptic phase, and the stage including the epileptic seizure, correspondingly.

an electric dipole or a more complicated system. Due to the crack strong wall vibration in the stage of the microbranching instability, it behaves as an efficient EM emitter. These EM precursors are detectable both at a laboratory and geological scale. Our main tool is the monitoring of the micro-fractures, which possibly occur in the pre-focal area before the final break-up, by recording their EM emissions. A multidisciplinary analysis in terms of fault modeling (Eftaxias et al., 2001), laboratory experiments (Eftaxias et al., 2002), criticality (Kapiris et al., 2004a; Contoyiannis et al., 2004, 2005), scaling similarities of multiple fracturing of solid material (Kapiris et al., 2004b), fractal electrodynamics (Eftaxias et al., 2004) and complexity (Kapiris et al., 2005a), seems to validate their association with the fracturing process in the pre-focal area.

Figure 1a shows the time series of the $10 \mathrm{kHz}$ magnetic field variations associated with the Athens EQ. We concentrate on this $\mathrm{kHz}$ EM activity observed before the 7 September 1999 Athens EQ $\left(38.2^{\circ} \mathrm{N}, 23.6^{\circ} \mathrm{E}\right)$ with a magnitude Ms $(A T H)=5.9$ at Zante station (Eftaxias et al., 2000, 2001, 2003, 2004; Kapiris et al., 2004). In terms of literature, this precursory emission has a strange, long duration (approximately a few days), thus it provides sufficient data for a statistical analysis. We note that the data were sampled at $1 \mathrm{~Hz}$.

\section{Electroencephalogram}

The brain is composed of very large numbers of non-identical non-linear neurons that are embedded in a vast and com- plex network. Electroencephalogram (EEG) provides a window, through which the dynamics of epilepsy preparation can be investigated. Epileptic seizures are abnormal, temporary manifestations of dramatically increased neuronal synchrony, either occurring regionally (partial seizures) or bilaterally (generalized seizures) in the brain. There is presently no standard mathematical model of EEG activity. Therefore investigators have been using various methods of signals analysis to describe stochastic and deterministic features of these signals. The prediction of epilepsy seizures plays an important role in epileptology (Litt and Javier, 2002), because we can propose a new diagnostic tool and a novel approach to seizure control based on this prediction (Iasemidis, 2003). Figure 1b shows a rat's epileptic seizure.

Adult Sprague-Dawley rats were used to study the epileptic seizures in EEG recordings. The rats were anaesthetized with an i.p. injection of Nembutal (sodium pentobarbital, $65 \mathrm{mg} / \mathrm{kg}$ of body weight), and mounted in a stereotaxic apparatus. An electrode was placed in epidural space to record the EEG signals from temporal lobe. The animals were housed separately postoperatively with free access to food and water, allow 2-3 days to recover, and handled gently to familiarize them with the recording procedure. Each rat was initially anaesthetized with a dose of pentobarbital $(60 \mathrm{mg} / \mathrm{kg}$, i.p.), while constant body temperature was maintained $\left(36.5-37.5^{\circ} \mathrm{C}\right)$ with a piece of blanket. The degree of anaesthesia was assessed by continuously monitoring the EEG, and additional doses of anesthetic were administered at the slightest change towards an awake pattern 

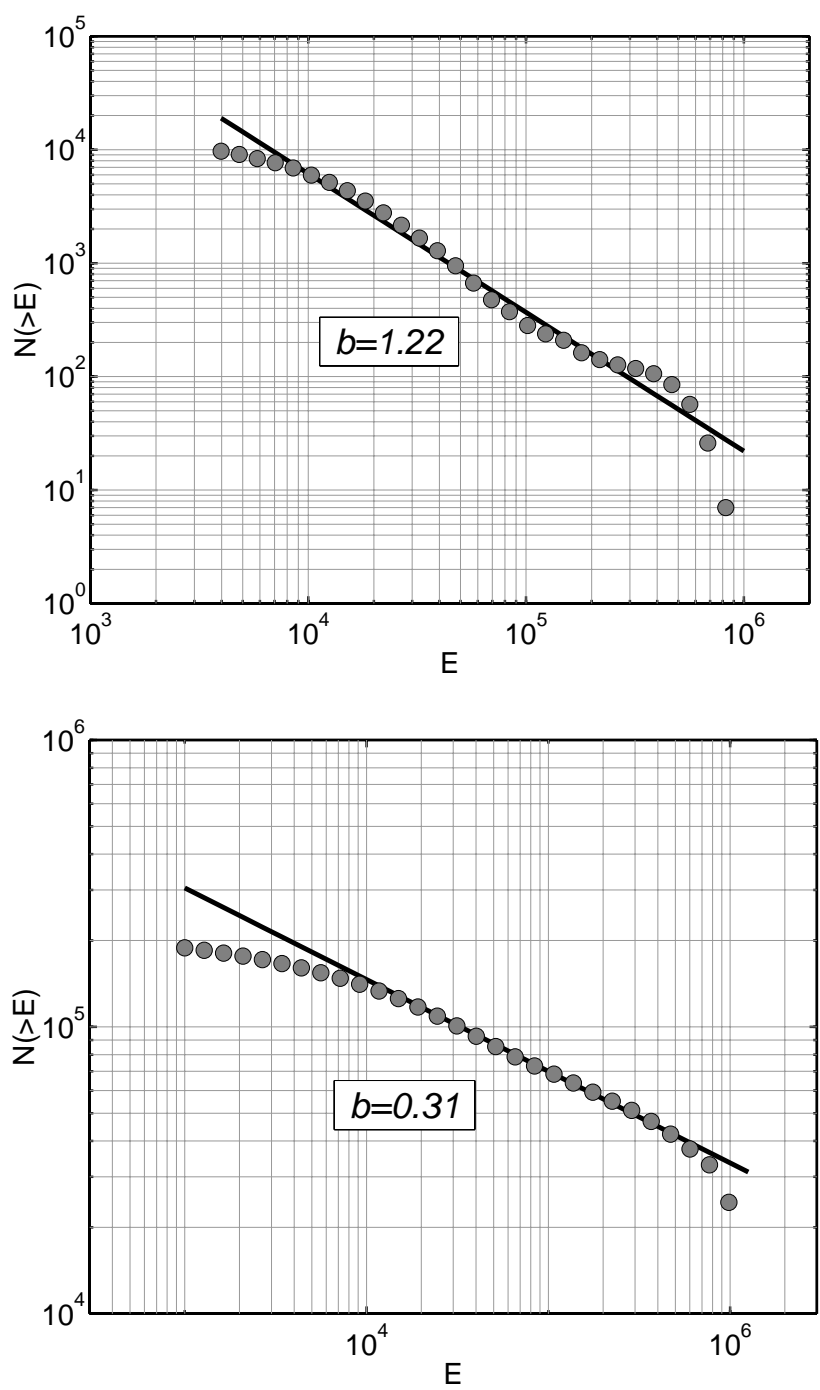

Fig. 2. Upper part. Number of electric pulses of the pre-ictal (red epoch in Fig. 1b) with energies higher than that given by the corresponding abscissa. The continuous line is the least squares fit of the power-law $N(>E)=E^{-b}$. Lower part. Number of electromagnetic pulses of the pre-seismic phase (red epoch in Fig. 1a) with energies higher than that given by the corresponding abscissa. The continuous line is the least squares fit of the power-law $N(>E)=E^{-b}$. The power-laws observed here is the footprint of a scale-free activity; an absence of characteristic lengths in the system.

(i.e., an increase in the frequency and reduction in the amplitude of the EEG waves). Then, bicuculline i.p. injection was used to induce the rat epileptic seizures. EEG signals were recorded using an amplifier with band-pass filter setting of $0.5-100 \mathrm{~Hz}$. The sampling rate was $200 \mathrm{~Hz}$, and the analogue-to-digital conversion was performed at 12-bit resolution. The seizure onset time is determined by visual identification of a clear electrographic discharge, and then looking backwards in the record for the earliest EEG changes from baseline associated with the seizure. The earliest EEG change is selected as the seizure onset time. The interval between the seizure onset time and injection time are considered as the maximum prediction duration or extended preictal phase.

\section{Fingerprints of scale free time and length behavior in the pre-seismic/pre-epileptic phase}

Complexity manifests itself in linkages between space and time, generally producing patterns on many scales and the emergence of fractal structure. A lot of work on complexity focuses on statistical power-laws, which describe the scaling properties of fractal processes and structures that are common among systems that at least qualitatively are considered complex. Scaling behavior or "scale-free" behavior means that no characteristic scales dominate the dynamics of the underlying process. Scale-free behavior reflects a tendency of complex systems to develop correlations that decay more slowly and extend over larger distances in time and space than the mechanisms of the underlying process would suggest (Bassingthwaihte et al., 1994; Barabasi and Albert, 1999; Bak, 1997). The emergence of a scale-free behavior is generally named "criticality" (Kadanoff et al., 1967). Thus, firstly we focus on the fundamental question whether power-law scaling behaviors in associated time series emerge as epileptic seizures or EQs approach.

\subsection{Focus on pre-seismic signals}

We have already studied this question in relation to preseismic EM emissions (Kapiris et al., 2004b) and, so far, find that the sequence of precursory EM pulses associated with the Athens EQ follow: (i) power-law distribution of the quiescent (waiting) times between successive EM pulses, i.e. $(\Delta t)^{-1.8}$; (ii) power-law distribution of the durations of the EM events, $(\Delta t)^{-1.9}$; (iii) a "Gutenberg-Richter type" distribution, namely, the cumulative number $N(>A)$ of EM pulses having amplitudes larger than $A$ follows the powerlaw $N(>A) \sim A^{-0.62}$.

These experimental results witness that the heterogeneous system in the focal area evolves to its global instability without characteristic time and length scales. This behavior implies the existence of an underlying critical dynamics.

Remark: Rabinovitch et al. (2001) have recently studied the fractal nature of EM radiation induced by rock fracture. The analysis of the pre-fracture EM time series reveals that the cumulative distribution function of the amplitudes follows the power $N(>A) \sim A^{-0.62}$. The accord of exponents in phenomena involving remarkably different scales should be considered as a further hint that the same fractoelectromagnetic critical dynamics holds from the geophysical scale down to the microscopic scale. 


\subsection{Focus on EEG time series}

We investigate the potential presence of power-law distributions in rat pre-epileptic phase (red epoch in Fig. 1b). The statistical analysis shows that the distribution of electric pulses of energy larger than $E, N(>E)$, is well fitted by the power-law $N(>E) \sim E^{-b}$, where $b=1.22$ (Fig. 2 upper part). The energy distribution of the binned data will follow the power-law $N(E) \sim E^{-\delta}$, where $\delta=(1+b)=2.22$ (Kapiris et al., 2004b). For comparison reasons, Fig. 2b (lower part) exhibits the number of EM events with energy $E$ higher than that given by the corresponding abscissa. The continuous line is the least square fit of the power law $N(>E) \sim E^{-b}$, where $b=0.31$.

The distribution of quiescent time between successive fluctuations, $l$, in EEG time series, is well fitted by the powerlaw $N(l) \sim l^{-\eta}$, where $\eta=1.15$ (Fig. 3 upper part). For comparison, in Fig. 3 (lower part), the same distribution is also presented for the pre-seismic EM time series (red epoch of Fig. 1a).

In summary, in the preparation stage of EQ (or epileptic seizure) the population of activated cracks (or firing neurons) in the focal area (or brain), seems to evolve through a non linear feedback mechanism triggering transitions between different metastable states, while, these transitions take the form of intermittent avalanche like events distributed without characteristic time and length scales.

\section{Emergence of common distinctive alternations in as- sociated scaling dynamical parameters as epileptic seizures and EQs approach}

The main feature of collective behavior is that an individual unit's (opening-crack or firing neuron) action is dominated by the influence of its neighbors; the unit behaves differently from the way it would behave on its own. Finally, ordering phenomena are emerged as the units simultaneously change their behavior to a common pattern (Vicsek, 2001, 2002). The emphasis in the structure formation in complex systems is bridging the gap between what one element does and what many of them do when they function cooperatively. Consequently, it follows that the science of complexity is about revealing the principles that govern the ways in which these new properties appear. Based on this background, we attempt to investigate not only the presence of power-law behavior in the pre-seismic (or pre-ictal) period, but mainly the temporal evolution of the associated scaling parameters as the main shock approaches. The visually apparent "patchiness" and evolution of the pre-seismic/pre-epileptic signals (Fig. 1) implies that different parts of time series have different scaling properties.

First, we focus on the statistics of the recorded series of fluctuations with respect to their amplitude, let's say $A\left(t_{i}\right)$. Any time series can exhibit a variety of autocorrelation struc-
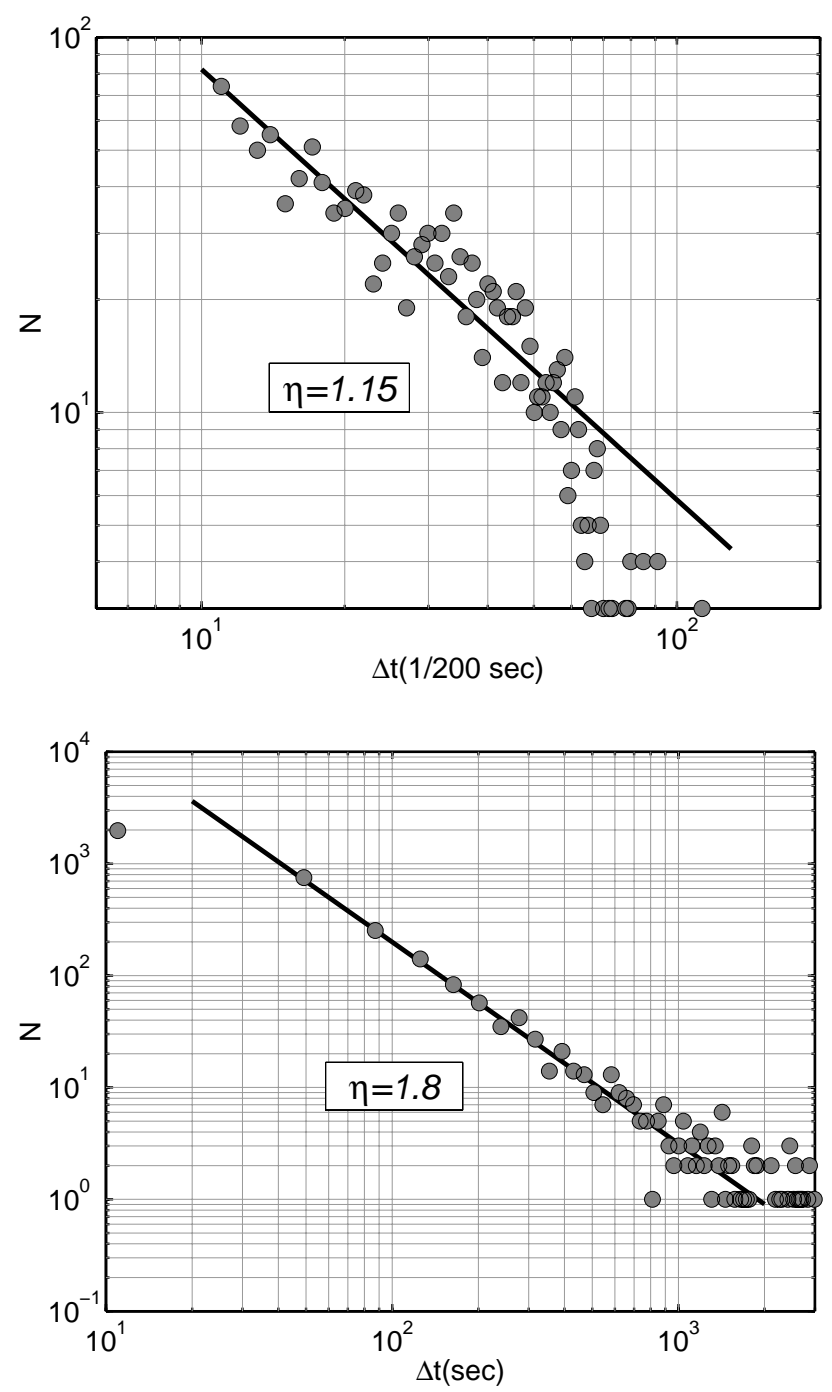

Fig. 3. Upper part. The distribution of the waiting times between two pre-epileptic electric pulses (red epoch in Fig. 1b). The straight line is the respective power-law. Lower part. The distribution of the waiting times between two pre-seismic electromagnetic pulses (red epoch in Fig. 1a). The strait line is the respective power-law. The occurrence of power-laws indicate that both systems develop critical correlations without characteristic time scales.

tures; successive terms can show strong (brown noise), moderate (pink noise) or no (white noise) correlation with previous terms. The strength of these correlations provides useful information about the inherent "memory" of the system. The power spectrum, $S(f)$, is probably the most commonly used technique to detect structure in time series. The quantity $S(f) d f$ is understood as the contribution to the total power from those components of the time series, whose frequencies lie in the interval between $f$ and $f+d f$. If the time series $A\left(t_{i}\right)$ is a self-affine time series that series cannot have a characteristic time scale. But a fractal time series cannot have any characteristic frequency either. The only possibility 

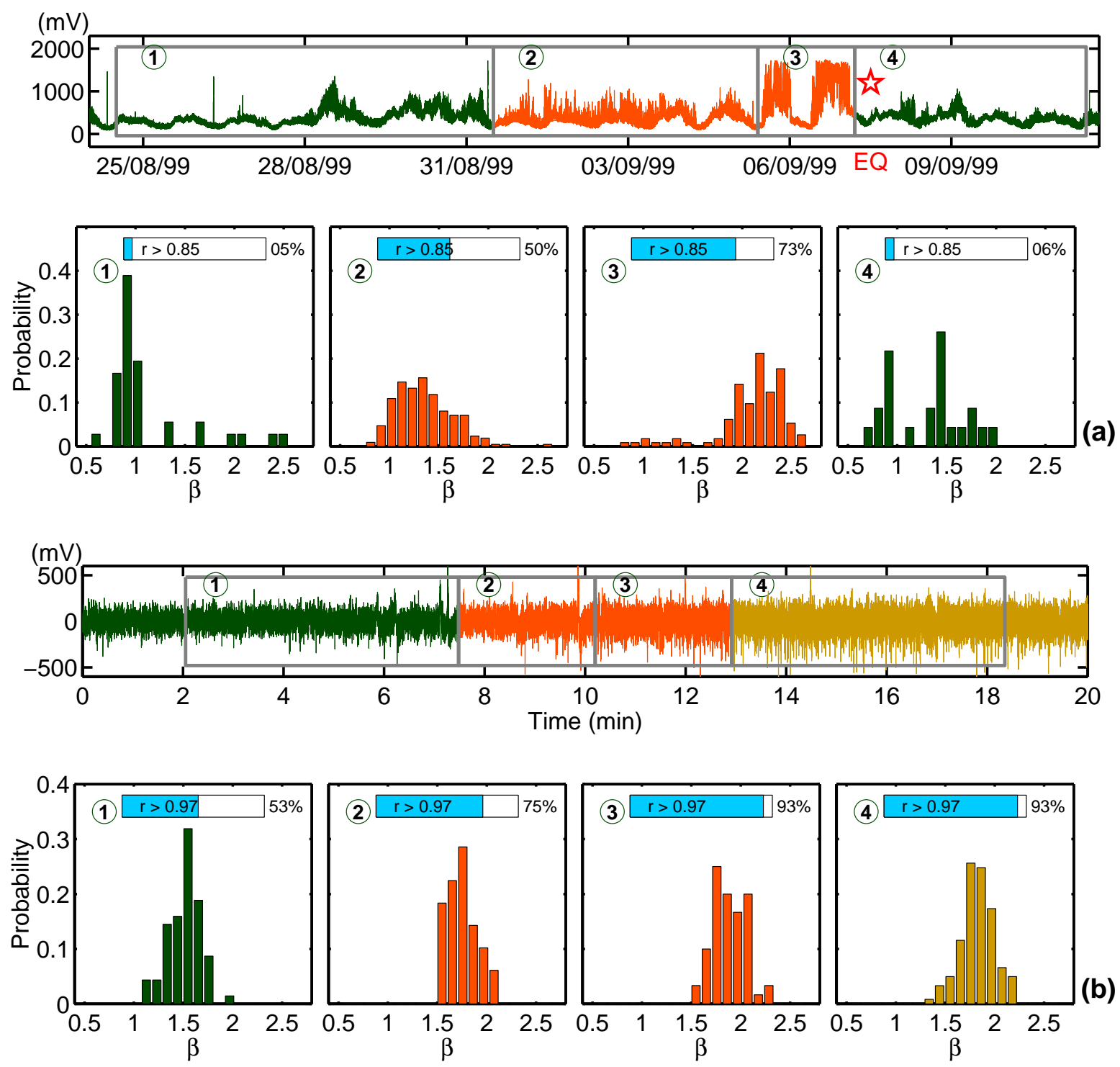

Fig. 4. Histograms of probability distribution of the exponent $\beta$ calculated on 1024 measurements segments for the four consecutive time intervals marked in accompanied time series plots. (a) refers to the pre-seismic EM pulses and (b) refers to the pre-ictal electric pulses. Insets show the percentage of segments with $r>0.85$ and $r>0.97$.

is then that the power spectrum $S(f)$ has a scaling form:

$$
S(f) \sim f^{-\beta}
$$

where the power spectrum $S(f)$ quantifies the correlations at the time scale $\tau \sim 1 / f$ and $f$ is the frequency of the Fourier transform. In a $\ln S(f)-\ln f$ representation the power spectrum is a line with spectral slope $\beta$. The linear correlation coefficient, $r$, is a measure of the goodness of fit to the powerlaw (1).

Our approach is to calculate the fractal parameter $\beta$ and the linear correlation coefficient $r$ of the power-law fit dividing the signal into successive segments of 1024 samples each, in order to study not only the presence of a power-law
$S(f) \sim f^{-\beta}$ but, as it was mentioned, the temporal evolution of these parameters.

The Continuous Wavelet Transform (CWT), using Morlet wavelet, is applied to compute the power spectrum, since being superior to the Fourier spectral analysis providing excellent decompositions of even transient, non-stationary signals (Kaiser, 1994). It has the ability of providing a representation of the signal in both the time and frequency domains. In contrast to the Fourier transform, which provides the description of the overall regularity of signals, the wavelet transform identifies the temporal evolution of various frequencies. This property suits the signals under investigation, because they 
are not stationary by their nature, and have a time varying frequency content.

\subsection{Information hidden in the temporal evolution of coeffi- cient correlation $r$}

Figures $4 \mathrm{a}$ and $\mathrm{b}$ present the temporal evolution of $r$ as the main geological/biological event is approached. We focus on the precursory epochs 2 and 3. We observe a significant increase of the correlation values, while, the percentage of $r$ that exceeds a certain threshold increases. At the tail of the precursory activity the fit to the power-law is excellent: a region with $r$ close to 1 is approached during the last stage of the pre-seismic/pre-seizure activity. The fact that the data are well fitted by the power-law (1) suggests that the preseizure (or pre-seismic) activity can be ascribed to a multitime-scale cooperative activity of numerous activated firingneurons/emitting-cracks. Here, an individual unit's behavior is dominated by its neighbors so that all units simultaneously alter their behavior to a common large scale fractal pattern. The gradual increase of $r$ indicates that the clustering of firing neurons/opening cracks in more "compact" fractal structures in brain/pre-focal area is strengthened with time.

We concentrate on the normal (quiescent) EM period (first epoch), preceding the emergence of the possible seismogenic EM anomaly (second and third epochs). We observe that the majority of segments $(\sim 95 \%)$ do not follow the power law (1). This means the EM background of the record does not behave as a temporal fractal. Thus the appearance of fractal structures seems to foretell on the launch of a possible seismogenic emission.

\subsection{Information hidden in the temporal evolution of scaling} spectral exponent $\beta$

Two classes of signal have been widely used to model stochastic fractal time series: fractional Gaussian noise (fGn) and fractional Brownian motion (fBm). These are, respectively, generations of white Gaussian noise and Brownian motion. The main difference between $\mathrm{fBm}$ and regular Brownian motion is that while the increments in Brownian motion are independent they are dependent in $\mathrm{fBm}$. The nature of fractal behavior (i.e., fGn versus $\mathrm{fBm}$ ) provides insight into the physical mechanism that generates the correlations: The fGn random fields describe fluctuations (additive noise) around mean state, while the $\mathrm{fBm}$ random model is derived from growth, deposition or random-walk processes.

The scaling exponent $\beta$ associated with the $\mathrm{fBm}$ model lies between 1 and 3 (Heneghan and McDarby, 2000). The range of $\beta$ from 0 to 1 indicates the fGn class. Figures $4 \mathrm{a}$ and $\mathrm{b}$ reveal that during the pre-ictal (or pre-seismic) phase (epochs 2 and 3 ) the $\beta$-values are distributed in the region from 1 to 3 . This observation implies that the associated part of both time series follow the fBm model.
Remark: We refer to the pre-seismic EM time series. It was mentioned that a very small number of segments $(\sim 5 \%)$ of the epoch 1 follows the power law (1). Their $\beta$-values are smaller than 1 , consequently, these segments belongs to the fGn class. This alteration further reveals the launch of the seismogenic emission from the background.

The distribution of $\beta$-exponents is also shifted to higher values (Figs. $4 \mathrm{a}$ and $\mathrm{b}$ ) during the precursory periods. This shift reveals several important features of the underlying mechanism. The fractal-laws observed corroborate to the existence of memory. The current value of the biological/geophysical signal co-varies not only with its most recent value but also with its long-term history in a scale-invariant, fractal manner, namely, the system refers to its history in order to define its future (Hausdorff and Peng, 1996). As the $\beta$-exponent increases the spatial correlation in the time series also increases. Consequently, the observed increase of $\beta$-values with time indicates the gradual increase of the memory, and thus the gradual reduction of complexity in the underlying dynamics.

Concerning epilepsy, the driving force behind a large number of studies on dimension analysis of EEG is that epileptic seizures are regarded as emergent states with reduced complexity compared to non-epileptic activity (Lai, 2002, and references therein): seizure onset represents a transition from relatively less orderly state to one of more orderly state (Iasemidis and Sackellares, 1996). Figure 4b also reveals that the epoch later in the epileptic seizure is of greater complexity than the early epoch. In summary, as the seizure approaches there is a transition from higher to lower complexity and then back to higher complexity prior to seizure termination.

The $1 / f$ observed power relationship implies that perturbations occurring at low frequencies can cause a cascade of energy dissipation at higher frequencies and that widespread slow oscillation modulate faster local events (Buzsaki and Draguhn, 2004). Indeed, Figs. 5a and b show that coherent fluctuations in a higher range of frequencies occur as the critical point is approached.

The observed color-type behavior of the power spectrum density $(\beta>0)$ means that the spectrum manifests more power at low frequencies than at high frequencies. If all frequencies are equally important, namely white noise, we have $\beta=0$. The increase, however, in the spectrum slope $\beta$ witnesses the gradual enhancement of lower frequency fluctuations, namely, the system selects to transmit more power at lower frequencies. These observations are consistent with the following physical picture: the activated micro-cracks/neurons interact and coalesce to form larger fractal structures, i.e., the events are initiated at the lowest level of the hierarchy, with the smallest elements merging in turn to form larger and larger ones. Concerning brain activity, higher frequency oscillations are confined to a small neuronal space, whereas very large networks are recruited during slow oscillations (Buzsaki and Draguhn, 2004, and 

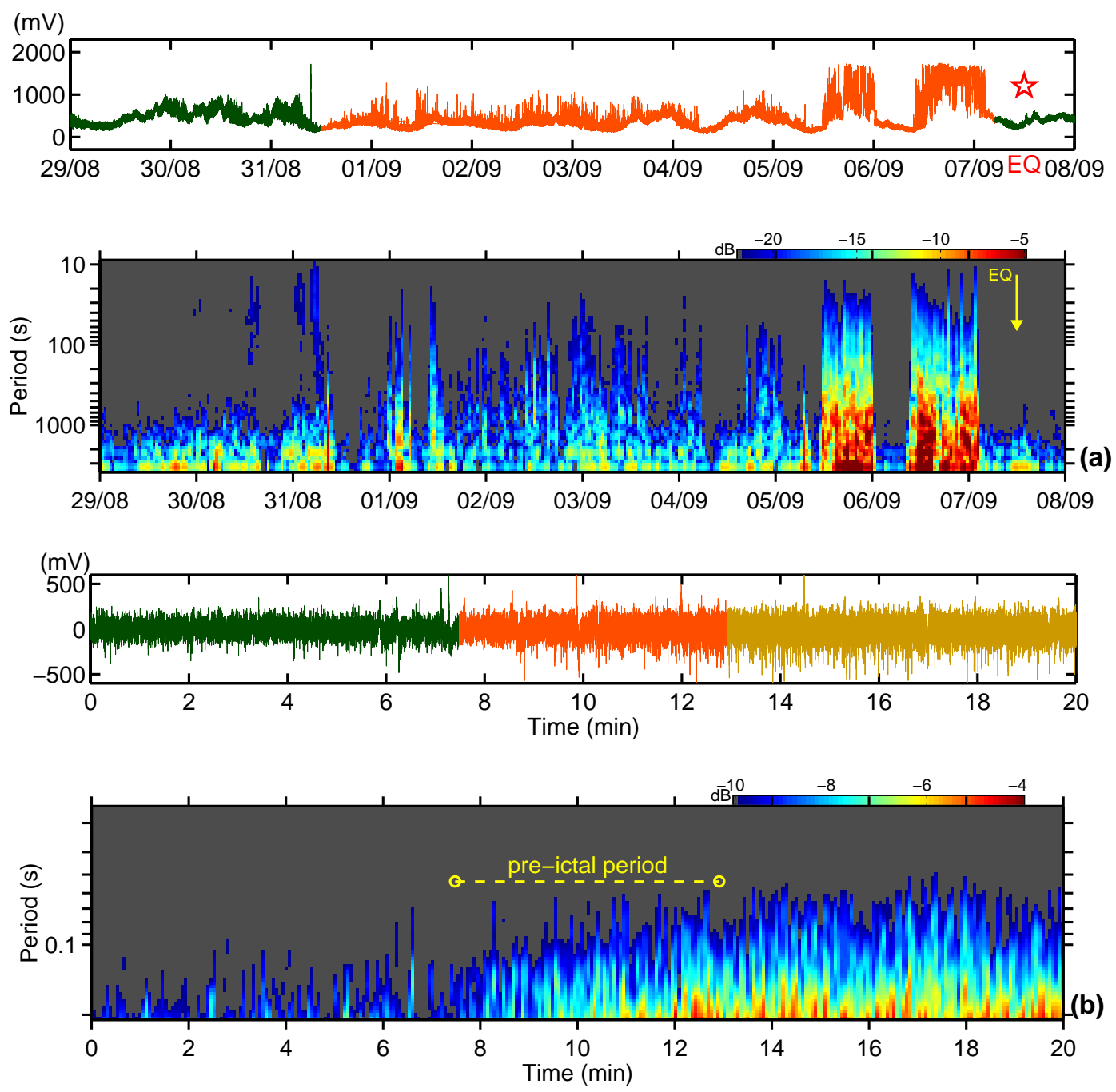

Fig. 5. The wavelet power spectrum of the time series associated with the Athens EQ (Fig. 1a) and the EEG time series including a rat epileptic seizure (Fig. 1b), correspondingly. The intensity scale on the top shows colour corresponding to the values of the square spectral amplitudes in arbitrary units.

references therein). There is only limited knowledge about seizure-generating mechanisms in humans. However, model simulations and animal experiments have led to the theory that seizure activity will be induced when a "critical mass" of neurons is progressively involved in synchronized discharging (Lehnertz and Elger, 1998).

Nature seems to paint the following picture: first, single isolated opening-cracks/firing-neurons emerge which, subsequently, grow and multiply. This leads to cooperative effects. Long-range correlations build up through local interactions until they extend throughout the entire system; finally, the main shock forms. The challenge is to determine the "critical epoch" during which the "short-range" correlations evolve into "long-range" ones. Figure 4 reveals that the closer the global instability, the larger the percentages of segments with $r$ close to 1 and the larger the shift of $\beta$ exponent to higher values; the $\beta$-values are maximal at the tail of the pre-seizure/pre-seismic state. This behavior reveals the emergence of a "critical epoch", namely, the transition to the epoch of synchronization of oscillating neuronal discharges (or fracto-EM oscillators).

\section{Monitoring the transition from anti-persistent to per- sistent behavior}

We attempt to shed more light on the physical significance of the systematic increase of the $\beta$-exponent during the preseismic/pre-ictal period. The $\beta$-exponent is related to the Hurst-exponent, $H$, by the formula (Turcotte, 1992)

$\beta=2 H+1$ with $0<H<1,, 1<\beta<3$

for the fractional Brownian motion (fBM) random field model (Henegham and McDardy, 2000). The exponent $H$ 

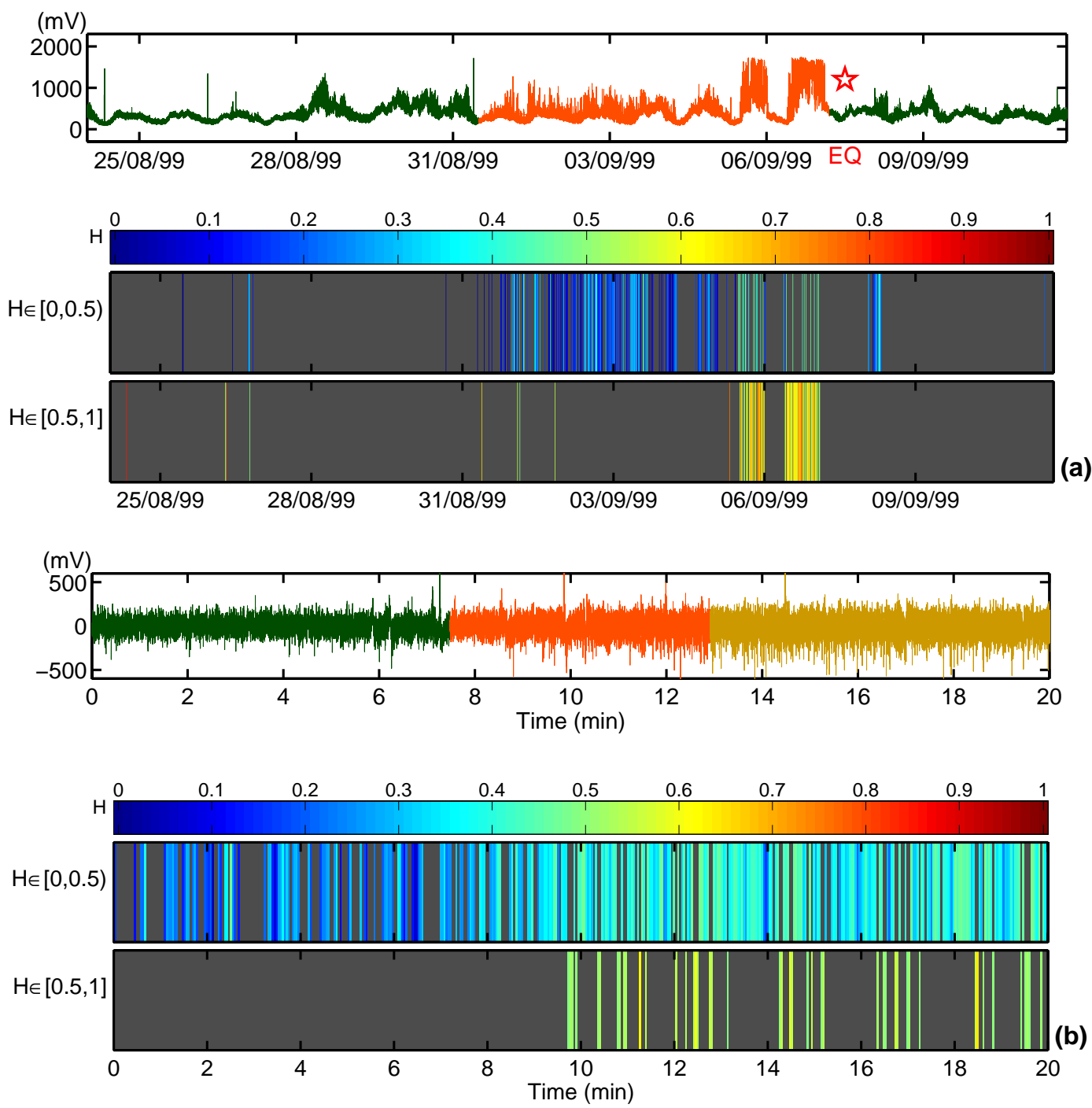

Fig. 6. Decomposition of time series into subsets, each characterized by a different local Hurst exponent $H$. We pay attention to the finding that the behavior of both the (a) pre-seismic signal and (b) pre-ictal signal become persistent in the tail of the precursory phase.

characterizes the persistent/anti-persistent properties of the signal according to the following scheme. At the limit $H=0$, the signal does not grow at all and is stationary. The range $0<H<0.5$ suggests an underlying anti-persistent (subdiffusion) behavior; namely, if the fluctuations increase in a period, it is likely to continue decreasing in the interval immediately following and vice versa. This leads to a very "noisy" variation. The range $0.5<H<1$ indicates a persistent (super-diffusion) behavior of the signal, i.e., if the amplitude of fluctuations increases in a time interval it is likely to continue increasing in the interval immediately following. $H=0.5$ suggests no correlation between the repeated increments. Consequently, the particular value $H=0.5$ takes on a special physical meaning: it signals the transition from antipersistent to persistent behavior in the time series.
Figure 6 reveals that the range $0<H<0.5(1<\beta<2)$ characterizes the initial phase of the pre-ictal (or pre-seismic) activity. Qualitatively, this anti-persistency reflects a set of fluctuations tending to induce stability in the system (Ivanov et al., 1999). The anti-persistent properties during this period imply the presence of a non-linear feedback mechanism triggering transitions between different metastable states, which, however, "kicks" the firing (or cracking) rate away from extremes in the brain (or pre-focal area). We pay attention to the systematic increase of the $H$-exponent during the precursory stage. This means that the non-linear negative feedback mechanism gradually loses its ability to "kick" the system away from extremes.

The appearance of persistent properties at the tail of the precursory period (Fig. 6) indicates that the geological/biological system has started to self-organize by a 
positive feedback process. This implies that the process acquires to a great degree the property of irreversibility, namely, the system is close to irreversible out of equilibrium phase transition. Consequently, the emergence of the persistent activity gives a significant hint of a considerable probability for a forthcoming global instability, namely, a significant seismic or epileptic event.

It is worth mentioning that laboratory experiments by means of acoustic EM emission verify that the main rupture occurs after the appearance of strong persistent behavior (Ponomarev et al., 1997; Lei et al., 2000, 2004).

\section{A key parameter: the order of heterogeneity}

In natural systems the units are not identical (Golomb and Rinzel, 1993). Most natural dynamical systems evolve in the presence of one or another type of disorder. Qualitatively, the presence of strong intermittency in the time series under study itself implies the effect of a highly heterogeneous regime, in which the heterogeneity occurs at many time-scales. Herz and Hopfield (1995) conclude that inhomogeneities are needed to generate a potentially rich connection between EQ cycles and neural reverberations. In the following, effects of heterogeneity on the dynamical systems under investigation are studied, in order to understand the cause of the transition from a purely anti-persistent to a purely persistent behavior.

Focus on fracture. In a highly disordered medium there are long-range anti-correlations, in the sense that a high value of threshold for breaking is followed by a low value, and vice versa (Sahimi and Arbadi, 1996). The anti-persistent character of the detected precursory EM emission reflects the fact that areas with a low threshold for breaking alternate with much stronger volumes. Crack growth continues until a much stronger region is encountered. When this happens, crack growth stops and another crack opens in a weaker region, and so on. In addition, the observed decrease of the anti-persistency can be understood if we accept that the micro-heterogeneity of the system becomes less anticorrelated with time.

On the other hand, in the case of a homogeneous medium, once a crack nucleates in the rock, stress enhancement at its tip is larger than at any other point of the medium, and therefore the next micro-crack almost surely develops at the tip (Sahimi and Arbadi, 1996). Thus, one expects to see long-range positive correlations, i.e., persistent behavior, $0.5<H<1$, in the emitted EM emission. Consequently, the bound $H=0.5$ witnesses the transition from a heterogeneous to an almost homogeneous regime.

The mentioned physical pictures can explain why the gradual decrease of heterogeneity leads to a decrease in the ability to drive the system away from a persistent mode, and thus, from an irreversible phase transition.
Focus on the EEG time series. Heterogeneity has been also introduced in the neuron intrinsic properties, e.g. Golomb and Rinzel (1993); Mousseau (1996). When a specific signal is input into brain, a particular location of cortex will respond (the membrane potential of response neurons increase). When the membrane potential of a neuron exceeds the threshold, the neuron sends out signals in the form of action potentials and then returns to the rest state (the neuron fires). The signal is transferred by the synapses to the other neurons, which has an excitatory or inhibitory influence on the membrane potential of the received cells according to whether the synapses are excitatory or inhibitory, respectively. The resulting potential, if it also exceeds the threshold, leads to the next firing step, and so on, giving an avalanche. Thus, a picture is emerged of dynamically evolving avalanches. The observed decrease of the antipersistency is also understood if we accept that the microheterogeneity of thresholds becomes less anti-correlated with time. The persistency in the tail of the pre-ictal epoch reveals the transition to an almost homogeneous regime. Golomb and Rinzel (1993) suggest that at low variability of thresholds, the network of neurons is still synchronized, while at large variability the system converges to a stationary asynchronous state. At intermediate variability, two regimes, characterized by a staircase-like dependence of the bursting rate on the cell excitability parameter, are seen. In one of them the network dynamics is synchronous and in the second it is anti-synchronous. A sharp transition between these two regimes, characterized by a discontinuity in the system order parameter, is observed as the level of disorder crosses a critical value. Corral et al. (1997) have also shown that diversity, introduced in terms of random distribution of natural periods, it is the mechanism that permits to observe selforganized criticality in the long time regime.

\section{The appearance of preferred direction of elementary activities just before the main shock}

The fractal dimension $d$ of the fBm time series is calculated from the relation $d=(5-\beta) / 2$ (or $d=2-H$ ) (Mandelbrot, 1982) which, after considering the shift of $\beta$-exponent (or $H$-exponent) to higher values, leads to a decrease of the fractal dimension as the biological (or geological) crisis is approached.

Note that a self-organized critical process, as the source of the temporal power-laws, would further suggest that similar power-laws exist also for parameters in the spatial domain (Linkenkaer-Hansen et al., 2001). Maslov et al. (1994) have formally established the relationship between spatial fractal behavior and long-range temporal correlations for a broad range of critical phenomena. By studying the time correlations in the local activity, they show that the temporal and spatial activity can be described as different cuts in the same underlying fractal. Characteristically, Mormann et 
al. (2003) have reported characteristic spatial and temporal shifts in synchronization that appear to be strongly related to pathological epileptic activity. On the other hand, authors have reported in-phase changes of the temporal and spatial Hurst exponents during sample deformation in laboratory acoustic/EM emission experiments (Ponomarev et al., 1997, and references therein). If we accept this consideration, the decrease of the fractal dimension reflects the appearance of a clear preferred direction of elementary activities just before the main shock. In general, the role of anisotropy in self-organized criticality has been highlighted (Pruessner and Jensen, 2003).

Focus on fracture. The terminal phase of fracture process is accompanied by a significant increase in localization and directionality (Eftaxias et al., 2004; Contoyiannis et al., 2005). The network of activated cracks becomes less ramified just before the main shock (Kapiris et al., 2004a).

Theoretical (Kolesnikov and Chelidze, 1985) aspects as indicate the following two major points: (i) elementary ruptures interact due to overlapping of their (scalar) stress field, giving rise to correlation effects, and (ii) at the same time the action of anisotropy inherent in the material leads to the existence of preferred directions in the arrangement of elementary ruptures.

On the laboratory scale, it is found that the spatial distribution of the hypocenters of the opening cracks ("laboratory EQs") shows fractal structure (Hirata et al., 1987). However, the fractal dimension significantly decreases with the evolution of the micro-fracturing process. Recent experiments further verify this behaviour (Lei et al., 2000, 2004).

As it was mentioned, Ponomarev et al. (1997) have reported in-phase changes of the temporal and spatial Hurst exponents during sample deformation in laboratory acoustic/EM emission experiments. Thus both correlation and anisotropy should be taken into account simultaneously (Chelidze, 1994).

Growing evidence suggests that rupture in strongly disordered systems can be viewed as a type of critical phenomenon (Gluzman and Sornette, 2001, and references therein). Experiments (Anifrani et al., 1995; Lamaignere et al., 1996; Garcimartin et al., 1997; Johansen and Sornette, 2000), numerical simulations (Sornette and Vaneste, 1992; Sahimi and Arbadi, 1996a; Sornette and Andersen, 1998) and theoretical aspects (Andersen et al., 1997) confirm this concept. Recently, we have shown that the anti-persistent phase of the precursory EM activity can be described as analogous to a second-order phase transition: from the phase of a sparse almost symmetrical random cracking distribution to a localized cracking zone that includes the backbone of strong asperities (Contoyiannis et al., 2005). A characteristic signature of the onset of a continuous (second-order) phase transition is symmetry breaking (S-B). This signature is hidden in the anti-persistent pre-seismic time series (Contoyiannis et al., 2005). The S-B here lies in the transition from non-directional uncorrelated to directional correlated crack growth. The completion of the S-B in the anti-persistent epoch signals that micro-fractures in the heterogeneous component of the pre-focal area, that surrounds the backbone of strong asperities on the fault plane, have finished: the "siege" of the backbone of asperities begins (Contoyiannis et al., 2005).

Consequently, the observed decrease of fractal dimension with time implies that the fracto-EM events have been restricted in the narrow zone including the backbone of strong large asperities that sustain the system (Eftaxias, 2004; Kapiris et al., 2004a). The emergence of strong persistent EM emission in the tail of the precursory emission reveals that the fracture of asperities has been started (Contoyiannis et al., 2005).

Focus on epileptic seizure. It has been reported that the characteristic abnormalities associated with epilepticseizures include simplification of the dendrite tree (Sackellares et al., 2000), which indicates that the network of firing neurons will be less ramified just before the next new seizure. This justifies a significant decrease of $d$-values with time in EEG time series just before the main event.

Usher et al. (1995) have presented a generic model that generates long-range (power-law) temporal correlations, $1 / f$ noise, and fractal signals in the activity of neural populations. Strong correlations are induced in the model by spontaneous symmetry breaking of the spatial pattern of activity across the network, leading to a preferred axis. This pattern is, however, metastable in the presence of noise: clusters of high activity diffuse throughout the system while undergoing strong internal fluctuations. The spatial pattern's persistent dynamics result in fractal (power-law) intervened interval distributions and $1 / f$ noise in the temporal dynamics. These features are in agreement with the results of the present study.

\section{Information hidden in the accelerating persistent stage of precursory activity}

Figures 5 and 7 reveal a significant accelerating energy release as the main geophysical or biological shock is approached. The observed acceleration implies that the two systems are not only near the "critical point" in the sense of having power-law correlations, but also in terms of exhibiting high susceptibility to perturbations. Due to the high level of the clustering of opening micro-cracks (or firing neurons), even a new small-activated cluster is able to connect other large clusters, and thus can generate a significant crisis. The emergence of persistency in the tail of the precursory emissions strongly encourages this hypothesis.

Concerning the EEG time series, the appearance of persistency just before the main shock indicates both: (i) the increase of the degree of excitability of the neural network (due to the increase of the average value of excitatory connections), and (ii) the increase of connectivity strength. A simulation study performed by Bondarenko and Ree Chay 

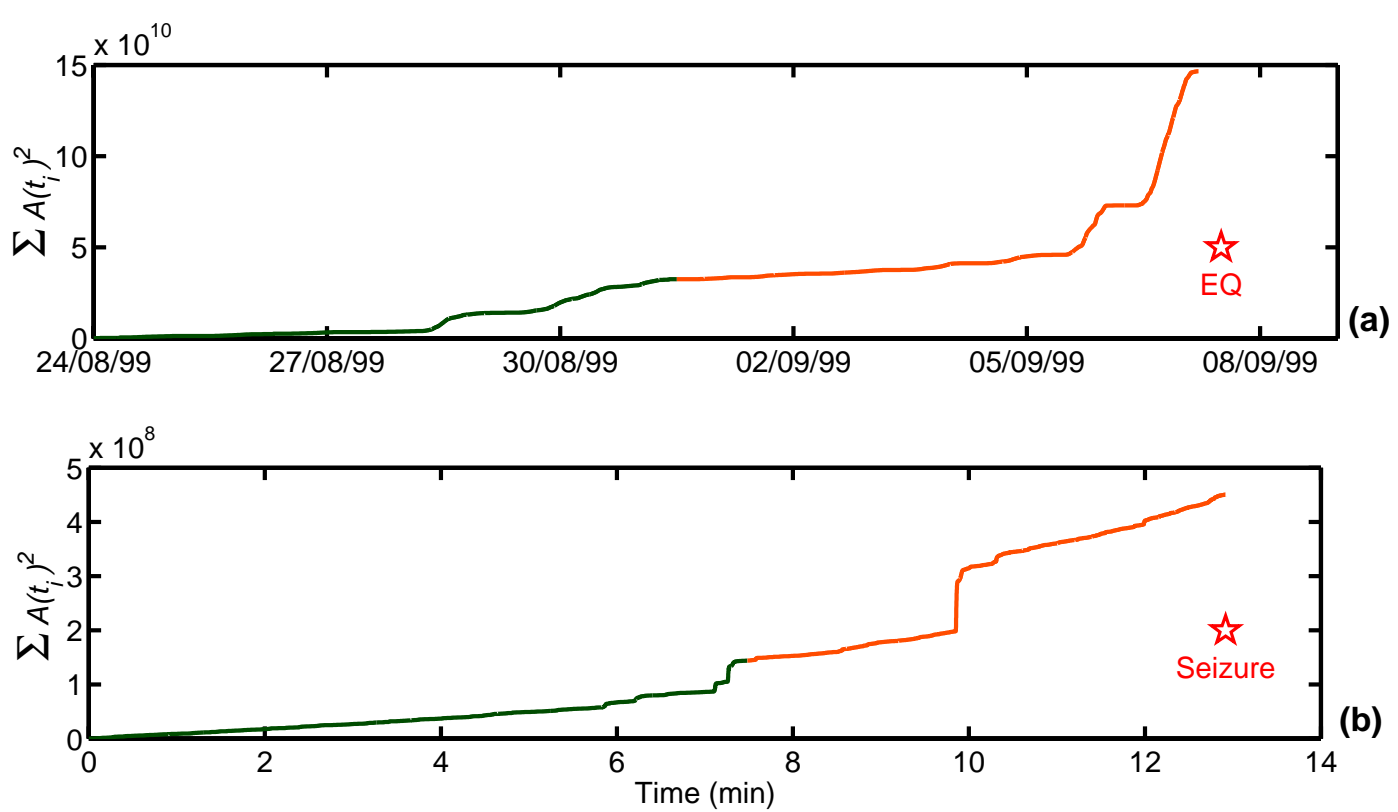

Fig. 7. The cumulative energy release, in arbitrary units, as a function of time: (a) for the pre-seismic EM time series (see Fig. 1a) and (b) for the EEG time series (see Fig. 1b).

(1998) suggests that the combination of these two actions leads to the synchronization of neurons outputs. Figure $5 \mathrm{~b}$ shows that new higher frequencies gradually emerge in the spectrum during the pre-ictal phase. The authors propose that the appearance of a high-frequency component allows a better synchronization and, as a consequence, an increase of the average activity amplitude. These suggestions are in agreement with the observed acceleration of energy release during the pre-ictal period. Bondarenko and Ree Chay (1998) conclude that these properties can be considered as the basis of the appearance of epilepsy.

The sensitivity of energy release and rich anisotropy provides two cross checking precursors for the impending catastrophic seismic or biological event.

\section{Footprints of an underlying self-synchronization}

Synchronization is one of the most fascinating non-linear phenomena appearing in a wide range of fields (Marodi et al., 2002, and references therein). The reduction of complexity with time in combination with the acceleration of energy release, the gradual reduction of anti-persistency and the emergence of persistent properties in the tail of the precursory epoch, are features consistent with the concept of forced synchronization (self-organization), which can be described qualitatively as follows. The population of opening cracks in the stage of the micro-branching instability (see section 2) can be considered as a source of radiated EM waves. Due to the interaction of these nonlinear oscillators, the energy exchange occurs. Gradually, due to internal relations, the energy interchange can result in synchronization of phases of oscillators. Finally, the radiation gradually becomes more coherent. During this process, the resulting EM energy grows considerably. Indeed, in the case of noncoherent radiation, namely if the phase distribution is chaotic and independent, the resulting energy $\mathrm{W}$ is proportional to the number of oscillators- $n$. On the contrary, in the case of full coherent radiation, the energy $\mathrm{W}$ is proportional to the square number of interacting oscillators- $n^{2}$ (Nicolis, 1986). We stress that if we accept the emergence of synchronization, then, the magnitudes of piezo-stimulated EM bursts can be influenced not only by the population of activated cracks but also can depend on the degree of coherency. Theoretical aspects as well as laboratory experience imply the appearance of synchronization in the final stage of EQ preparation process.

Aksenov and Lokajicek (1997), based on the comparison of laboratory and field observations, have shown the existence of symptoms of forced synchronization (spectral simplification and transformation into a narrow spectral band) and coherency of radiation in seismic foci.

Chelidze and Lursmanashvili (2003), based on experiments on the spring-slider system subjected to a constant pull and superimposed with weak mechanical or electromagnetic periodic forces, have recently shown that at definite conditions the systems manifests synchronization of micro-slip events with weak excitation. The regimes of slip vary from the perfect synchronization of slip events (acoustic emission) with the perturbing periodic mechanical or EM impact, to the complete desynchronization of micro-slip events and perturbations. 
Gil and Sornette (1996) have shown a general correspondence between SOC and synchronization of threshold oscillators. Recently, theoretical studies imply that the phenomenon of synchronized chaos also appears in fundamental models for EQs (de Sousa Vieira, 1999; Akishin et al., 2000; Tsukamoto et al., 2003). Characteristically, Akishin et al. (2000) have presented an extension of the BurridgeKnopoff (BK) model of EQ dynamics, one of the basic models of theoretical seismicity. The extension is based on the introduction of non-linear terms for the inter-block springs of the BK model. Their analysis reveals synchronization of the collective motion and produces stronger seismic events. We pay attention to the fact that for the BK system the Hurst exponent is much greater than 0.5 . It lies between 0.6 and 0.8 , stabilizing about 0.7 , when time increases. This value, which indicates persistency, is very close to the values of Hurst exponents obtained in the tail of the pre-seismic EM activity under investigation, as well as for real EQs that is also about 0.7 (Lomnitz, 1994).

We turn our attention to epileptic seizures. In general, to produce a voltage change large networks of neurons have to be active synchronously (Buzsaki and Draguhn, 2004).

The epileptic seizure models exhibit distinct collective properties that range from systemwide synchronization to self-organized criticality: phase locking without global synchronization has been identified as an important borderline phenomenon between both cases and has been observed under various conditions (Herz and Hopfield, 1995, and references therein).

Numerous of studies suggest that neuronal hypersynchrony underlies seizures (Lehnertz and Elger, 1995; Bondarenco and Ree Chay, 1998; Gong et al., 2003; Buzsaki and Draguhn, 2004). Non-linear analyses have suggested that the seizure onset represents a transition from the interictal period (the period between seizures) to one of increased synchronous activity, and that a more orderly state characterizes an epileptic seizure (Iasemidis and Sackellares, 1996).

The reduction of complexity with time in EEG time series, the acceleration of energy release, and the emergence of persistent properties in the tail of the pre-ictal epoch are features consistent with the concept that the epileptic seizures are episodic events resulting from abnormal synchronous discharges from cerebral neuronal networks.

Buzsaki and Draguhn (2004) concluded that "An important function of the brain is the prediction of future probabilities. Feedforward and feedback networks predict well what happens next. Oscillators are very good predicting when”.

\section{A possible common scenario for the development of severe epileptic and seismic shock}

By monitoring the temporal evolution of the fractal spectral characteristics in electroencephalograms and pre-seismic EM emissions we find that common distinctive alterations in the associated scaling parameters indicate a transition from the low-activity (normal) state to an abnormal (high-activity) state (major EQ/epileptic seizure), as follows: (i) Appearance of memory effects, namely, emergence of long-range correlations. This implies a multi-time-scale cooperative activity of numerous activated sub-units. (ii) Increase of the spatial correlation in the time series with time. This indicates a gradual transition from a less orderly state to a more orderly state. (iii) Gradual increase of the number of time intervals with "critical fractal characteristics". It indicates that the clustering of activated units in more "compact" fractal structures strengthened with time; the self-organized complexity becomes more obvious. (iv) Predominance of large events as the main biological or geophysical shock is approached. This observation signals that the precursory events are initiated at the lowest level of the hierarchy, with the smallest elements merging in turn to form larger and larger ones. (v) Appearance of strong anti-persistent behavior in the first epoch of the pre-ictal and pre-seismic activity. It implies the existence of a non-linear negative feedback mechanism that "kicks" the system away from extremes. (vi) Decrease of the antipersistence behavior with time. It implies that the mentioned non-linear negative feedback mechanism gradually loses its ability to "kick" the system away from extremes. (vii) Appearance of persistency in the "tail" of the precursory activities. This gives a hint that the system acquires to a great degree the property of irreversibility. (viii) Significant acceleration of the energy release as the main shock approaches. It shows that the system exhibits high susceptibility even to a small perturbation. (ix) Decrease of the fractal dimension of the time series with time. This suggests the establishment of preferred directions in elementary activities. (x) Gradual appearance of higher frequencies in the spectrum with a simultaneously increase of the amplitudes at each emission rate as the shock approaches, mainly characterizing lower emission rates. The coherent fluctuations at all scales further indicate that the critical point is approached.

The aforementioned crucial precursory footprints (including temporal alterations in associated scaling parameters) may indicate the following common scenario for the development of a severe epileptic or seismic shock. During the first epoch of the transition from the normal state to the abnormal one, the neuronal (or fracture) mechanism of patient (or strained focal area) is in self-organized complexity. The energy is released spasmodically in avalanches with a selfsimilar size distribution, however, with a restricted and systematically fluctuating correlation length. The system is in a sub-critical anti-persistent state, in which case the external input signal can only access a small, limited part of the system. Long-range correlations gradually build up through local interactions until instabilities extend throughout the entire system. The scale over which the interacting units are correlated sets the size of the largest event that can be expected at that time. Such instabilities can channel the brain/focal area into a globally nonequilibrium super-critical state. Weak 
events can be the agents by which longer correlations are established. A population of weak events will advance the correlation length by an amount depending on the brain/focal area state, triggering intense shocks only if the condition is right. In a sub-critical anti-persistent regime, a population of small activated events leads to a quickly decaying activity. In the super-critical persistent state is just able to continue "indefinitely". This naturally explains why while brain/focal area not being close to criticality can exhibit only a small response to a small perturbation, its response near criticality to this same small perturbation may become explosive due to the very high "susceptibility". An intense shock destroys long correlations, creating a new normal period during which the process repeats by rebuilding correlation lengths towards criticality and the next large event. Thus a large epileptic or seismic shock can act as a sort of "critical point" dividing the epileptic/seismic cycle into a period of growing correlations before the great event and a relatively uncorrelated phase after.

Concerning the brain, this view is consistent with Bak's view (1997). Bak argued that the brain can be neither subcritical nor super-critical but self-organized critical. In the first case, the external input signal can only access a small limited part of the information. In the second case, any input would cause an explosive branching process within the brain, and connect the input with essentially everything that is stored in the brain. Except in last case, the brain has an appropriate sensitivity to small shocks (Zhao and Chen, 2002).

Remark: The precursory symptoms indicate that the proposed method of analysis is interesting for readers belonging to different scientific fields. Major magnetospheric disturbances are undoubtedly among the most important phenomena in space physics and also a core subject of space weather. They are relatively rare events: as in the case of atmospheric storms, EQs, solar flares, etc., the occurrence of geomagnetic storms rapidly decreases as their magnitude grows. Magnetic storms occur when the accumulated input power from the solar wind exceeds a certain threshold. MS intensity is usually represented by an average of the geomagnetic perturbations measured at four mid-latitude magnetometer stations (http://swdcwww.kugi.kyoto-u.ac.jp/). The resulting geomagnetic index Dst is a widely used measure of the symmetric ring current during MSs. During MSs the complex system of the Earth's magnetosphere, which corresponds to an open (input - output) spatially extended nonequilibrium system, manifests itself in linkages between space and time, producing characteristic fractal structures. We have shown (Balasis et al., 2005) that the above mentioned distinctive alterations in scaling parameters of Dst index time series occur as a strong magnetic storm approaches. The increase of the susceptibility coupled with the transition from anti-persistent to persistent behavior indicates that the onset of a severe magnetic storm is imminent. This evidence enhances the universal character of the precursory symptoms under study.

\subsection{Footprints of an underlying intermittent criticality}

Pure SOC models imply a system perpetually near global instability, hence reducing the degree of predictability of individual events (Bak et al., 1987; Bak and Tang, 1989). However, natural systems very rarely break down without presenting time-dependent effects. Thus, these simplified models fail to reproduce some important properties of the precursory spatiotemporal clustering in real systems (Grasso and Sornette, 1998; Main and Al-Kindy, 2002). Characteristically, in a recent Letter, Yang et al. (2004) conclude that "EQs are unlikely phenomena of SOC; therefore further research on EQ prediction should not be discouraged or opposed by the argument that EQs are SOC and as a result unpredictable". Authors, in order to bridge both, the hypothesis of an underlying self-organized critical state and the occurrence of precursory phenomena analyze more complex and realistic models (Sornette and Sammis, 1995; Sammis et al., 1996; Heimpel, 1997; Huang et al., 1998, Hainzl et al., 2000). In this direction, the school of "intermittent criticality" predicts a time-dependant variation in the activity as the "critical point" is approached, implying a degree of predictability. The aforementioned evolution incorporates a time dynamics with memory effects, which is overall characterized as intermittent criticality.

Particularly, this hypothesis predicts two different precursory phenomena in space and time, namely, the acceleration energy release and the growth of the correlation length. In terms of intermittent criticality the acceleration of energy release is the consequence of the growth of the spatial correlation length. Thus, a large shock which is not immediately preceded by a period of accelerating energy release represents a system which had previously reached a critical state but has not yet had a large event to perturb the system away from the critical state. On the other hand, an accelerating activity which is not followed by a large shock mirrors a system which has achieved criticality but in which a large event has not nucleated.

The present study suggests that it can be important to distinguish between SOC and intermittent criticality in the study of the seismic/epileptic cycle. A proper recognition and understanding of tuning parameters leads to the development of improved models having higher performance reliability. One of the main features in the complexity is the role that the topological disorder plays in such systems. The range of size scales characterizing heterogeneities of the thresholds might be acted as a tuning parameter of the underlying final dynamics.

We note that the preparation of a major magnetic storm can be also studied in terms of "Intermittent Criticality". 


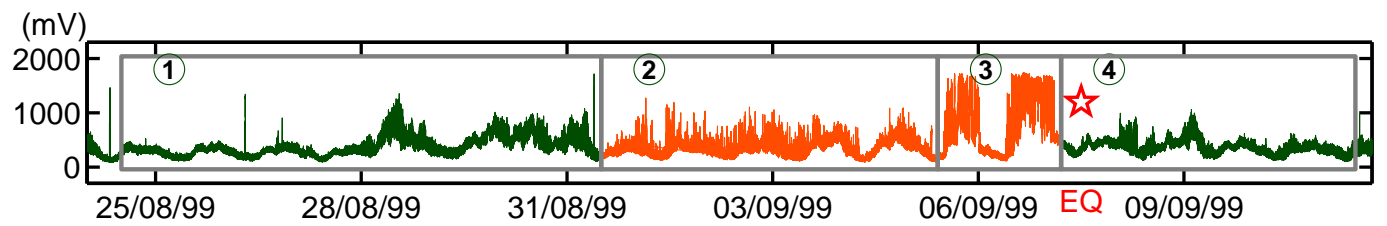

(1)

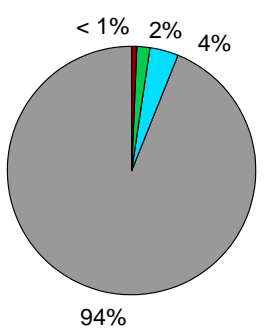

(2)

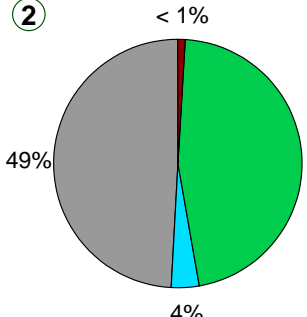

(3)

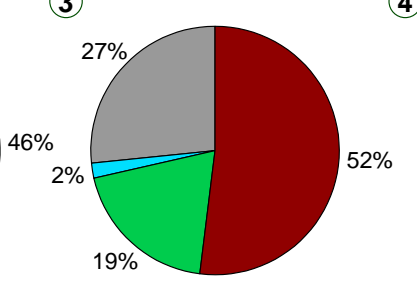

(4)

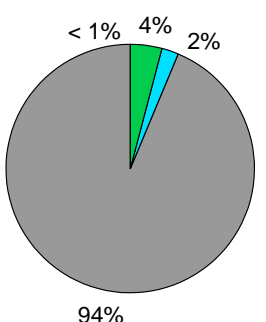

(a)
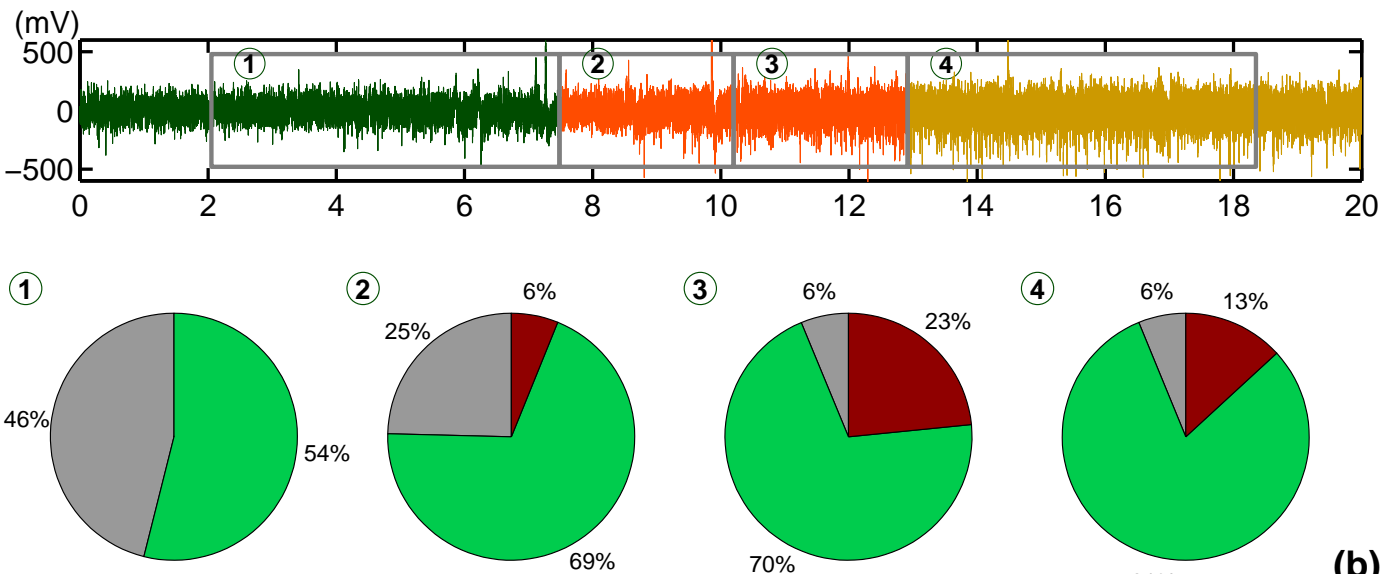

(3)

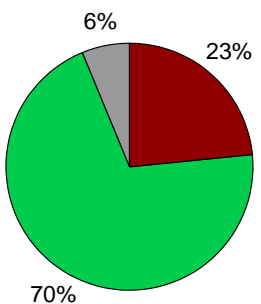

(4)

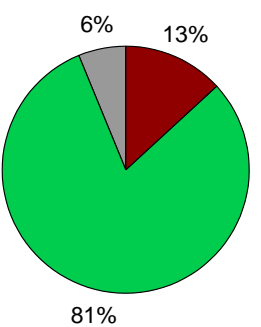

Fig. 8. The grey sector shows the percentage of the time in each epoch where the time series does not follow a power law of the type $S(f) \sim f^{-\beta}$. The green and red sectors demonstrate the percentage of the time in each epoch where the time series follow the fBm model exhibiting anti-persistent and persistent behavior, respectively. The light blue sectors reveal the percentage of the time in each epoch where the time series follow the fGn model. This figure suggests that a reduced complexity of brain activity (lower part) or pre-focal area activity (upper part) coupled with the appearance of $\mathrm{fBm}$ persistent behavior, as soon as it is of sufficient duration, can be regarded as a specific feature defining states which proceed to an epileptic seizure or EQ.

\section{Is the evolution towards global instability inevitable after the appearance of distinctive symptoms?}

A question that arises is whether the evolution towards global instability of the system is inevitable after the appearance of distinctive footprints in the pre-catastrophic time series. The transition from anti-persistency to persistency and the significant acceleration of the energy release, namely, the increase of the susceptibility of the system, the extension of fluctuations to new frequency scales with simultaneous predominance of large events and the emergence of strong anisotropy, i.e. the appearance of preferential direction, indicates that the generation of a very intense seismic/epileptic event becomes unavoidable.

Our analysis reveals an interesting transition from the antipersistent to the persistent regime. The anti-persistent behavior characterizes the brain/pre-focal area during weak events, while the catastrophic events are in reasonable agreement with persistent models. It is important to bear in mind that the Democratic Fiber Bundle Model (DFBM) for fracture exhibits an interesting transition as a function of the amplitude of the disorder. As the disorder decreases, there exists a tricritical transition (Andersen et al., 1997) from a progressive damage (second-order) regime to a Griffith-type abrupt rupture (first-order) regime, where rupture occurs without significant precursors. The corresponding pictures in each regime are not in contradiction.

Contoyiannis et al. (2002) have introduced the intermittent dynamics of critical fluctuation (IDCF) method of analysis for the critical fluctuations in systems which undergo a continuous phase transition at equilibrium. This method is based on a previous work (Contoyiannis et al., 2000) according to which the fluctuations of the order parameter at the critical point of a continuous phase transition obey an intermittent 

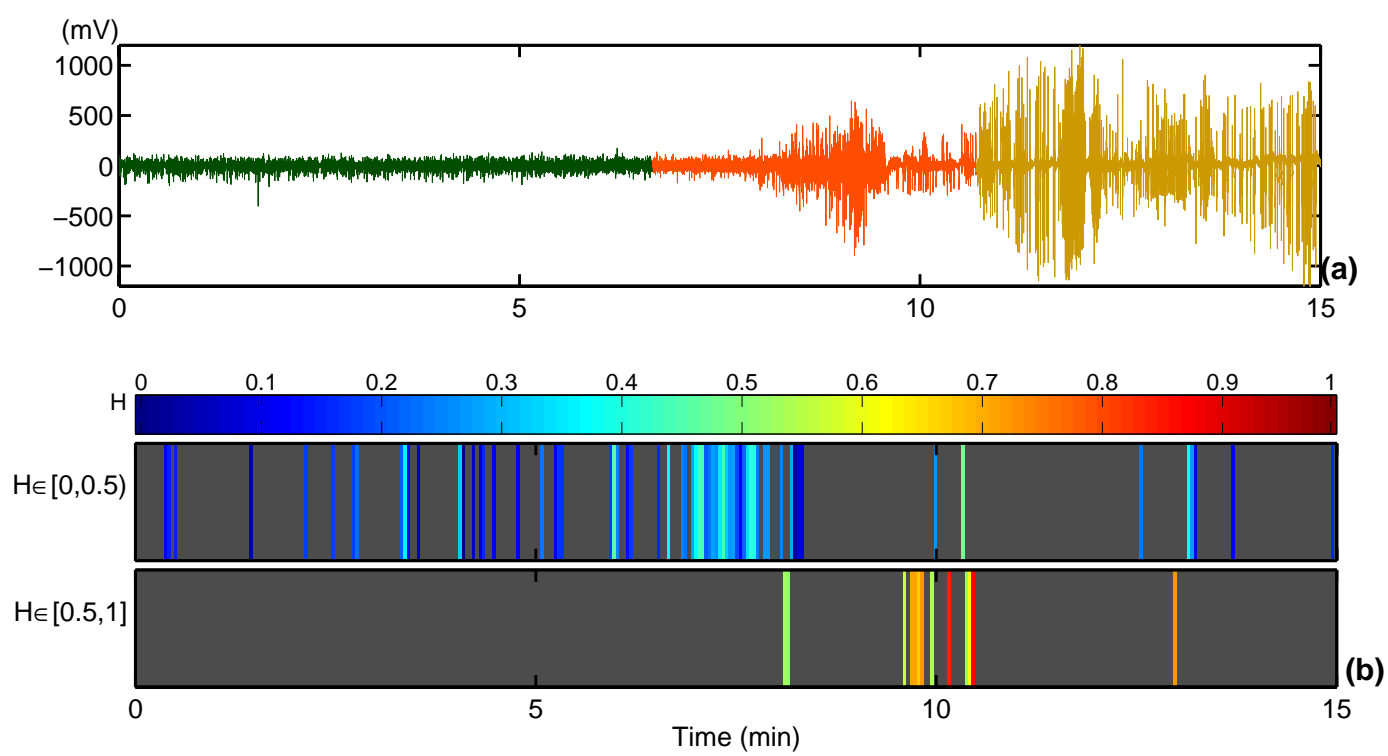

Fig. 9. (a) Rat EEG time series. The sampling rate was $200 \mathrm{~Hz}$. Bicuculline i.p. injection was used to induce the rat epileptic seizures. The green, red and ochre epochs show the normal state, the pre-epileptic phase, and the stage of the epileptic seizure, correspondingly. (b) Decomposition of time series into subsets, each characterized by a different local Hurst exponent $H$. The behavior of the pre-ictal signal becomes persistent in the tail of the precursory phase.

dynamics which can be analytically expressed by a one dimensional map of intermittency type I. Recently, the focal area has been modelled by (i) the backbone of strong and almost homogeneous large asperities that sustains the system, and (ii) the strongly heterogeneous medium that surrounds the family of strong asperities (Contoyiannis et al., 2005). The analysis of pre-seismic EM signals in terms of the IDCFmethod (Contoyiannis et al., 2005) suggests that the initial anti-persistent part of the EM precursor is triggered by fractures in the highly disordered heterogeneous component that surrounds the essentially homogeneous backbone of asperities, and described in analogy with a thermal phase transition of second order. On the contrary, the persistency in the tail of the emission is thought to be due to the fracture of the high strength asperities if and when the local stress exceeds their fracture stress, and it is a purely out of equilibrium process. The abrupt emergence of strong VLF emission in the tail of the precursory EM radiation, showing persistent behavior, is regarded as a fingerprint of the local dynamic fracture of asperities in the focal zone, namely, faulting nucleation (Contoyiannis et al., 2005). This concept is strongly supported by seismological observations, information obtained from radar interferometry, and space-time Thermal Infrared Radiation (TIR)-signals (Kapiris et al., 2005b). In addition, laboratory studies indicate the appearance of persistent properties in the acoustic emission during the fracture of asperities (Lei et al., 2000, 2004).

Figures $8 \mathrm{a}$ and $\mathrm{b}$ verify that a reduced complexity of brain (or pre-focal area) activity coupled with the appearance of $\mathrm{fBm}$ persistent behavior, as soon as it is of sufficient duration, can be regarded as a specific feature defining states, which proceed to an epileptic seizure or EQ.

Figure 9 refers to a new example of rat epilepsy. The analysis further supports the concept that the transition to persistent regime can be considered as a candidate precursor. We refer to the first event included in preparation (red) epoch of the seizure (Fig. 9). This abnormal event constitutes a kind of "foreshock" of the ensuing generalized epileptic seizure. We think that the emergence of the main (generalized) seizure results from the further increase of the correlation length and the appearance of persistent properties of sufficient duration in the EEG time series.

\section{Extracting precursory symptoms in terms of non- linear techniques}

The performed linear fractal spectral analysis suggests as main result that the last phase of $\mathrm{EQ/epileptic} \mathrm{seizure} \mathrm{prepa-}$ ration process mischaracterized by a clear transition from higher to lower complexity. In the sequel, we verify this result in terms of non-linear technique: before the $\mathrm{EQ} /$ seizure symptom appeared, the complexity had begun declining.

\subsection{Non-linear analysis in terms of T-complexity}

A computable complexity measure, such as T-complexity, gives evidence of state changes leading to the point of global fracture (Karamanos et al., 2005).

The T-complexity, which has been described in details elsewhere (Tichener, 1998, 1999) is defined along similar 

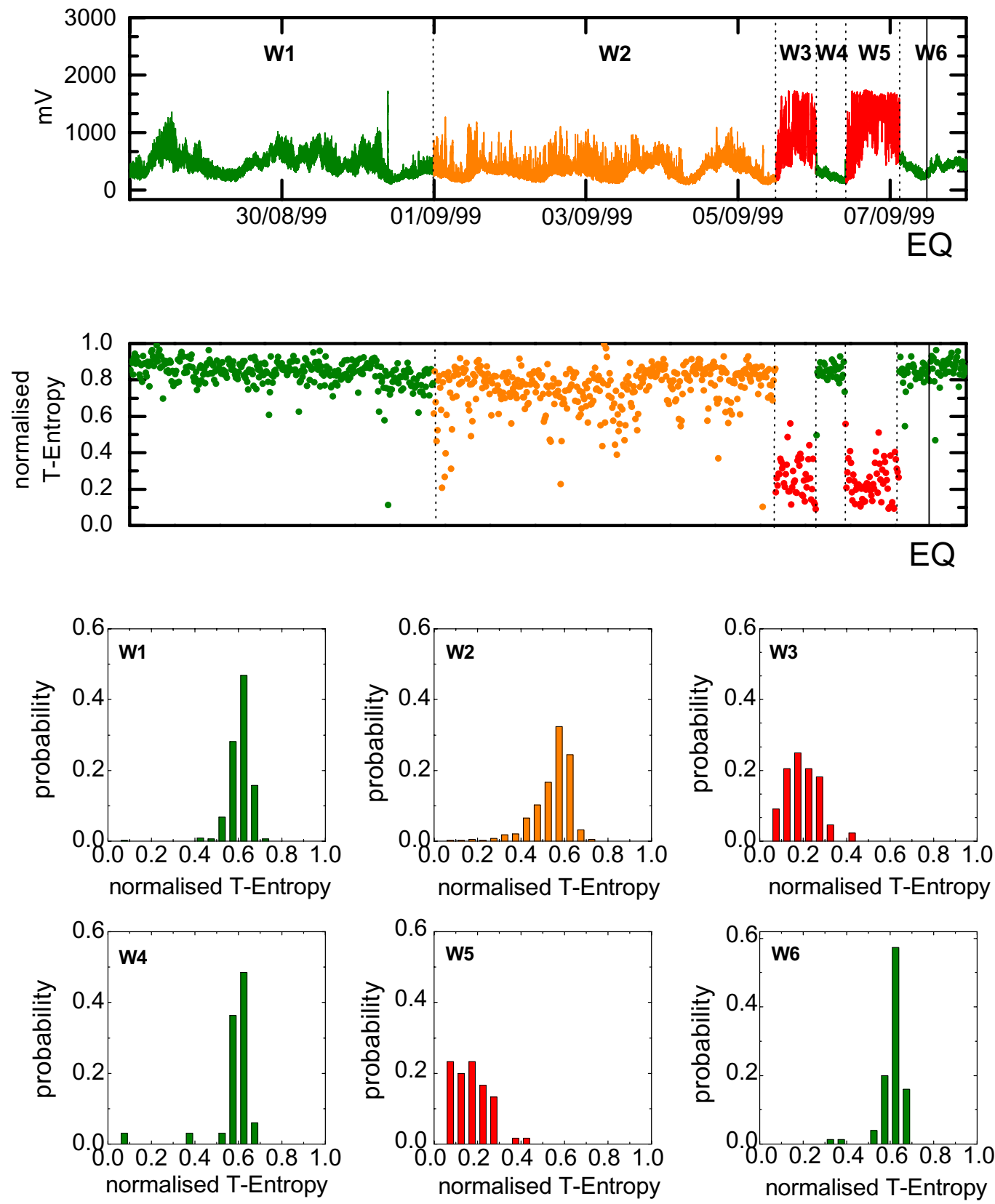

Fig. 10. (a) We distinguish six epochs (W1-W6) in the evolution of the EM signal associated with the Athens EQ. (b) Each point denotes the normalized T-entropy calculated by considering consecutive time windows of 1024 points for the six epochs defined in (a). We observe an important decrease of the T-entropy values during the emergence of the two strong impulsive emissions at the tail of the precursory activity (windows W3 and W5). (c) Histograms of the probability distribution of the normalized T-entropy, calculated for the six epochs. We also observe a significant reduction of the complexity as the main event is approaching, in particular during the epochs W3 and W5.

lines to the Lempel Ziv production complexity (Lempel and Ziv, 1976), which measures the generation rate of new patterns along a digital sequence, but a recursive hierarchical pattern copying algorithm (Tichener, 2000) is used. A characteristic example of an actual calculation for a finite string is given in Ebeling et al. (2001). T-complexity serves as a measure of complexity of the signal: the lower the value of T-complexity, the more "ordered" it is. Dividing the time series into sub-series, we study how the T-entropy evolves as we go from one sub-series to another. The aim is to discover a clear difference of dynamical characteristics as the catastrophic event is approaching. 
Application to the Athens EQ. We first divide the EM signal in six consecutive epochs depicted in (Fig. 10a). For each of these six epochs, we calculate the T-entropy associated with successive segments of 1024 samples each (Fig. 10b). Thereafter we study the distributions of T-entropy values in these windows (Fig. 10c).

We underline the similarity of the distributions of the Tentropy values in the first, fourth and sixth time intervals. This almost common distribution characterizes the order of complexity in the background noise of the EM time series. The associated high T-entropy values indicate a strong complexity. We turn our attention to the second and third time intervals, namely during the emergence of the precursory emission. We observe a significant decrease of the T-entropy values. This evidence reveals a strong reduction of complexity in the underlying fracto-electromagnetic mechanism during the launching of the two strong EM bursts. This finding might be indicated by the appearance of a new phase at the tail of the EQ preparation process, which is characterized by a higher order of organization. Sufficient experimental evidence seems to support the association of the aforementioned two EM bursts with the nucleation phase of the impending EQ (Eftaxias et al., 2000; Kapiris et al., 2005b; Contoyiannis et al., 2005).

Application to the EEG signal. We first divide the signal in six consecutive epochs depicted in Fig. 11a. For each of these six epochs, we calculate the T-entropy associated with successive segments of 1024 samples each (Fig. 11b).

Thereafter, we study the distributions of T-entropy values in these windows (Fig. 11c). As the seizure approaches there is a transition from higher to lower T-entropy values: we observe a significant decrease of the T-entropy values prior to shock. Higher degrees of synchronization are reflected in lower signal complexity. After seizure initiation, but still relatively early in the seizure, the signal is one of relatively low complexity. As the seizure evolves, the complexity of the signal gradually increases. The observed increasing signal complexity during seizure evolution is consistent with progressive de-synchronization of the seizure activity.

In summary, the analysis in terms of T-complexity reveals a significant reduction of complexity prior to an important $\mathrm{EQ} /$ epileptic seizure. We pay attention to the fact that the epoch of high complexity corresponds to that of the antipersistent behavior, while the epoch of low complexity corresponds to that of the persistent behavior. The corresponding pictures in each regime are not in contradiction.

\subsection{Non-linear analysis in terms of Correlation Dimen- sion and Approximate Entropy}

Other computable complexity measures, namely, correlation dimension $D_{2}$ and approximate entropy ApEn, have been recently used in pre-seismic EM time series (Nikolopoulos et al., 2004) and epileptic seizure time series data (Radhakrishnan and Gangadhar, 1998; Lehnertz and Elger, 1998)
The correlation dimension $D_{2}$ was first established by Grassberg and Procaccia (1983) and is based on the Takens theorem (Takens, 1981). Generally, the correlation dimension represents the independent degrees of freedom that are required for the proper description of a system or for the construction of its model. A time series that results from a complex non-linear dynamic system yields a larger value for the correlation dimension; a time series, which results from a regular and linear dynamic system, shows lower correlation dimension values.

Entropies indicate the variety of the patterns which are included in an ensemble or a certain time interval. In particular, ApEn is a new statistical parameter derived from the Kolmogorov-Sinai entropy formula which quantifies the amount of regularity in data. Intuitively, one reason that the presence of repetitive patterns of fluctuation in a time series render it more predictable than a time series in which such patterns are absent. ApEn reflects the likelihood that "similar" patterns of observations will not followed by additional "similar" observations. A time series containing many repetitive patterns has a relatively small ApEn; a less predictable, namely more complex, process has a higher ApEn. In a perfectly regular data series the knowledge of the previous values enables the prediction of the subsequent value. The approximate entropy value would be zero. For example, in a perfectly regular data series $0,0,1,0,0,1, \ldots$, knowing that the two previous values were 0 and 0 enables the prediction that the subsequent value will be 1 . With increasing irregularity, even knowing the previous values, the prediction of the subsequent value will get worse. The approximate value will increase.

Focus on fracture. The main results obtained from our study of the EM time series associated with the Athens EQ in terms of correlation dimension and approximate entropy (Nikolopoulos et al., 2004) are summarized in Fig. 12. We underline the almost common distributions of the $D_{2}$-values and ApEn-values in the first, second and fourth time intervals (Fig. 12). This almost common distribution refers to the background noise of the EM time series. The associated high $D_{2}$-values and ApEn-values indicate a strong complexity. We observe a significant abrupt decrease of the $D_{2}$ values and ApEn-values as we move to the third time window, namely, during the emergence of two impulsive signals. This reveals a strong loss of complexity in the underlying fracto-EM activity.

Focus on epileptic seizures. Lehnertz and Elger (1998) have evaluated the capability of non-linear series analysis in terms of effective correlation dimension to extract features from brain electrical activity predictive epileptic seizures. Time-resolved analysis of human EEG time series indicates a significant loss of complexity prior to and during seizures (the related effective correlation dimension decreases up to 2 ), while, far away from any seizure, the associated effective correlation dimension varies around 9. 

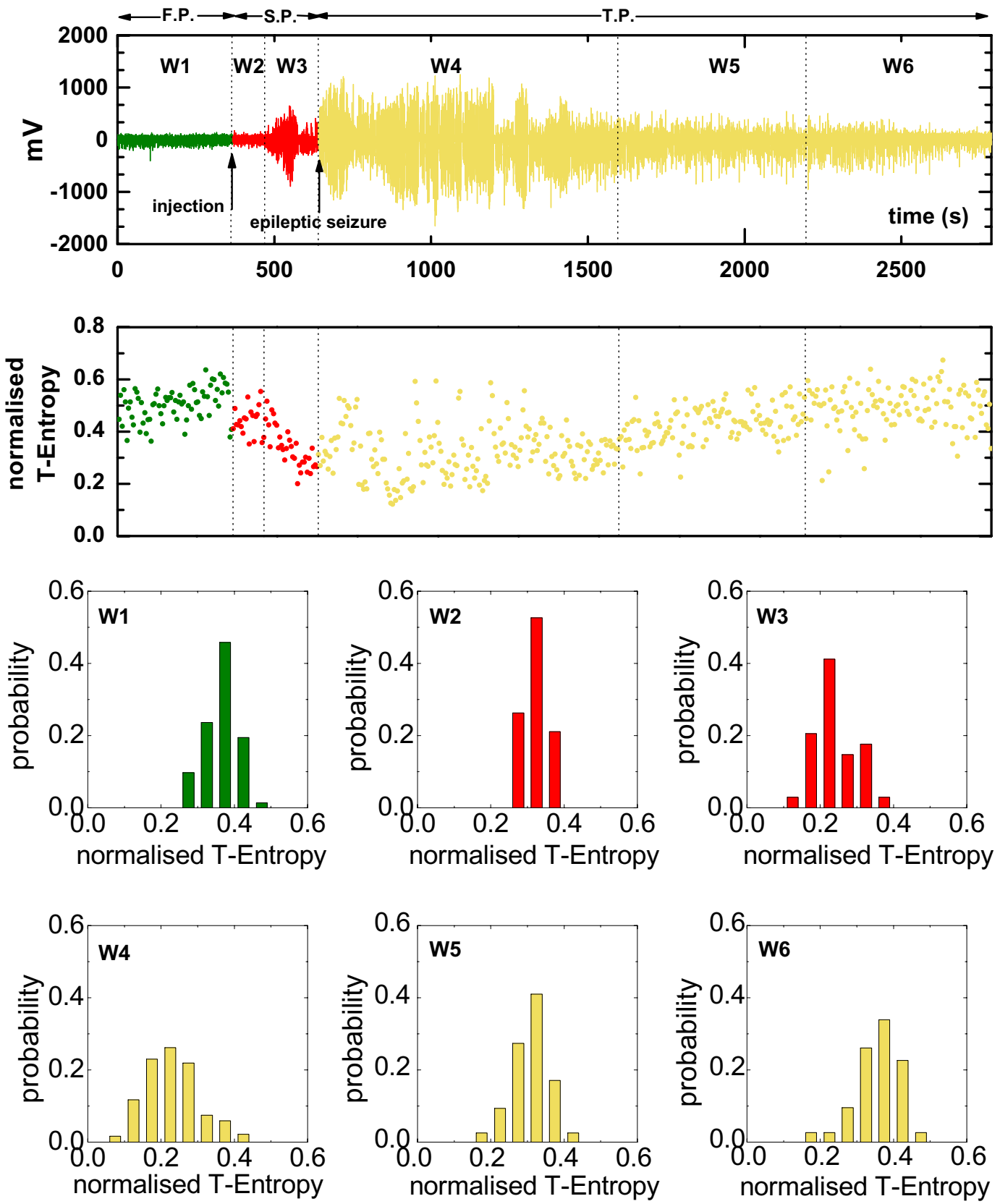

Fig. 11. First row: We distinguish six epochs (W1-W6) in the evolution of the EEG signal associated with a rat epileptic seizure. Second row: Each point denotes the normalized T-entropy calculated by considering consecutive time windows of 1024 points. We observe an important decrease of the T-entropy values during epochs 2 and 3 at the tail of the pre-epileptic epoch. Third-fourth row: Histograms of the probability distribution of the normalized T-entropy values, calculated for the six epochs. We also observe a reduction of the complexity as the epileptic seizure is approaching, in particular during the epochs W2 and W3.

Radhakrishnan and Gangadhar (1998) have used the ApEn to quantify the regularity embedded in the seizure time series data. They found that the seizure becomes more regular and coherent in the middle part.

We conclude that the analysis in terms of the correlation dimension and approximate entropy also corroborates the appearance of a new phase in the tail of the EQ/epileptic seizure preparation process, which is characterized by a higher order of organization.

The convergence between non-linear and linear analysis provides a more reliable detection concerning the emergence of the last phase of the EQ preparation process. More precisely, we claim that our results suggest an important principle: significant complexity decrease, and accession of 

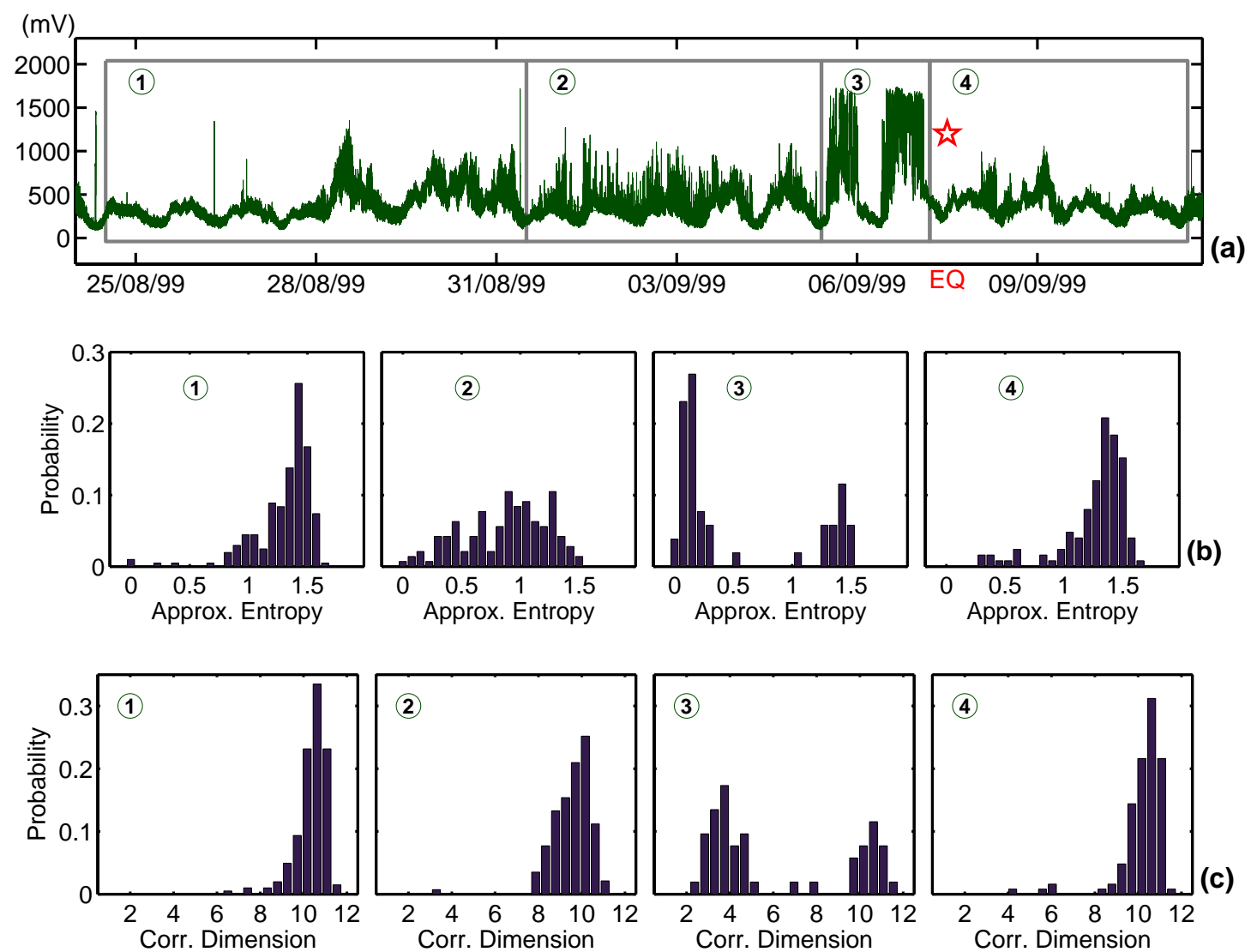

Fig. 12. (a) $10 \mathrm{kHz}$ magnetic field variations prior to the the Athens EQ. (b, c) Correlation Dimension $\left(D_{2}\right) /$ Approximate Entropy $(A p E n)$ estimations in consecutive segments of 3000 samples each. We trace the distribution of these $D_{2} /$ ApEn values for four consecutive epochs depicted in (a). Epochs 1 and 4 correspond to the EM quiescence that precedes and follows the EM activity. The almost similar distribution in epochs 1 and 4 characterize the EM background (noise). In epoch 2 the little deformation of the distribution to the left in respect to the one of the pure noise indicates that the initial part of the precursory emission is characterized by a little reduction of the complexity in respect to the high complexity of the pure noise. The right lobe that appears in epoch 3 corresponds to the EM background, while the left lobe corresponds to the EM precursory activity. We observe a dramatic shift of the distribution of the $D_{2} /$ ApEn values in epoch 3 . This evidence indicates a strong reduction of complexity during the emergence of the two strong EM bursts in the tail of the precursory emission.

persistency in time series can be confirmed at the tail of the pre-catastrophic epoch, which can be used as diagnostic tools for the impending shock.

\section{Conclusions}

Recently, mathematical techniques have been developed that are useful for quantifying and describing the complex and chaotic signals. Whereas much research in this area has been conducted in relation to the physiologic systems (cardiac system, brain activity), we show that the same methods also promise in relation to the pre-fracture EM activity.

The existence of precursory features in pre-seismic EM emissions is still in discussion. We bear in mind that, in principle, it is difficult to prove associations between events separated in time, such as EQs and their EM precursors. Complexity suggests a striking similarity in behavior close to irre- versible phase transitions among systems that are otherwise quite different in nature. Interestingly, theoretical studies suggest that the EQ dynamics at the final stage and neural seizure dynamics should have many similar features and can be analyzed within similar mathematical frameworks.

EEG time series provide a window through which the dynamics of shock preparation can be investigated under wellcontrolled conditions, as well as, in the absence of noise. Consequently, the analysis of a pure pre-epileptic time series will help in establishing a reliable collection of criteria to indicate the approach to the biological shock. In this study we highlight the precursory symptoms emerging in approaching epileptic seizures, and their resemblance to those precursory symptoms occurring prior to an EQ.

We observe that both kinds of catastrophic events follow common behavior in their pre-catastrophic stage. This evidence supports the hypothesis that the detected EM anomaly is originated during the micro-fracturing in the pre-focal 
area. More precisely, in terms of non-linear analysis, i.e. Tcomplexity, correlation dimension and approximate entropy, a transition from a regime of high complexity to a regime of low complexity indicates the approach to both biomedical and geological shock. In terms of an independent linear analysis, namely, a fractal spectral analysis, a transition from an anti-persistent regime to a persistent regime reveals the approach to the main shock. We stress that the transition from the regime of high complexity to the regime of low complexity coincides temporary with the transition from the anti-persistent regime to the persistent regime. Thus, the coherent results suggest a significant loss of complexity in combination with emergence of persistency in the tail of the pre-catastrophic epoch. The collection of common precursory signatures in the pre-seismic/pre-epileptic time series not only enhances the consideration that the detected EM anomaly has been originated during the final stage of the EQ preparation process, but also specifies the temporal distance from the shock. The dynamical parameters: T-complexity, correlation dimension $\left(D_{2}\right)$, approximate entropy (ApEn), and Hurst exponent $(\mathrm{H})$, seem to represent a measure of the distance of the system from the global instability. The lower the T-complexity values, the lower the $D_{2}$-values, the lower the ApEn-values, and the higher the Hurst-values are, then, the smaller the distance of the system from the "critical point" (global instability).

Acknowledgements. We gratefully acknowledge financial support by the European Union program EPEAEK/PYTHAGORAS $70 / 3 / 7357$.

Edited by: M.-C. Llasat

Reviewed by: two referees

\section{References}

Akishin, P., Altaisky, M., Antoniou, I., Budnik, K., and Ivanov, V.: Burridge-Knopoff model and self-similarity, Chaos, Solitons and Fractals, 11, 207-222, 2000.

Aksenov, V. and Lokajicek, T.: Influence of nonlinear processes in seismic foci on released energy, Pure and Applied Geophysics, 149, 337-355, 1997.

Al-Kindy, F. and Main, I.: Testing self-organized criticality in the crust using entropy: A regionalized study of the CMT global EQ catalog, J. Geophys. Res., 108(B11), 5/1-5/9, 2003.

Andersen, J., Sornette, D., and Leung, K.: Tricritical behavior in rupture induced bydisorder, Phys. Rev. Lett., 78, 2140-2143, 1997.

Anifrani, J-C., Floc'h, C., and Souillard, D.: Universal log-periodic correction to renormalization group scaling for rupture stress prediction from acoustic emissions, J. Phys. I France, 5, 631-638, 1995.

Bak, P., Tang, C., and Weisenfeld, K.: Self-organized criticality: an explanation of 1/f noise, Phys. Rev. A, 38, 364-374, 1987.

Bak, P. and Tang, C.: Earthquakes as a self-organized critical phenomenon, J. Geophys. Res., 94, 15 635-15 637, 1989.

Bak, P.: How nature works, Oxford UP, 1997.
Balasis, G., Vassiliadis, D., Kapiris, P., Mandea, M., and Eftaxias, K.: From normal state to magnetic storms in terms of fractal dynamics, EGU05-A-01767, NH9.09/NP4.06-1TH3O-005, 2005a.

Balasis, G., Kapiris, P., and Eftaxias, K.: From normal state to magnetic storms in terms of fractal dynamics, arXiv:physics/0411157, 2004.

Balasis, G., Kapiris, P., and Eftaxias, K.: Similarities in Precursory Features in Strong Seismic Shocks and Magnetic Storms, Geophys. Res. Abstr., 8, 02649, 2006.

Barabasi, A. and Albert, R.: Emergence of scaling in random networks, Science, 286, 509-512, 1999.

Bassingthwaihte, J., Liebovitch, L., and West, J.: Fractal physiology, Oxford UP, New York, 1994.

Bondarenko, V. and Ree Chay, T.: Desynchronization and synchronization processes in a randomly coupled ensemble of neurons, Phys. Rev. E, 58, 8036-8039, 1998.

Buzsaki, G. and Draguhn, A.: Neuronal oscillations in cortical networks, Science, 304, 1926-1929, 2004.

Buzsaki, G. and Traub, R.: Physiological basis of EEG activity, in: Epilepsy: a comprehensive textbook, p. 819-830, edited by: Engel, J. and Pedley, T., Philadelphia, PA, Lippincott Raven, 1997.

Chang, T., Vevedensky, D., and Nicoll, J.: Differential renormalization-group generators for static and dynamic critical phenomena, Phys. Rep., 217-279, 1992.

Chelidze T. and Lursmanashvili, O.: Electromagnetic and mechanical control of slip: Laboratory experiments with slider system, Nonlin. Processes Geophys., 20, 1-8, 2003.

Chelidze, T., Reuschle, T., and Gueguen, Y.: A theoretical investigation of the fracture energy of heterogeneous brittle materials, J. Phys. Condens. Matter., 6, 1857-1868, 1994.

Chen, Y., Ding, M., and Scottt Kelso, J.: Long memory processes (1/f type) in human coordination, Phys. Rev. Lett., 79(22), 4501-4504, 1997.

Contoyiannis, Y., Diakonos, F., Kapiris, P., Peratzakis, A., and Eftaxias, K.: Intermittent dynamics of critical pre-seismic electromagnetic fluctuations, Phys. Chem. Earth, 29, 397-408, 2004.

Contoyiannis, Y., Kapiris, P., and Eftaxias, K.: Monitoring of a preseismic phase from its electromagnetic precursors, Phys. Rev. E, 71, 066123-1-066123-14, 2005.

Corral, A., Perez, C., Diaz-Guilera, A., and Arenas, A.: Selforganized criticality and synchronization in a lattice model of Integrate-and-Fire oscillators, Phys. Rev. Lett., 74, 118-121, 1995.

Corral, A., Perez, C., and Diaz-Guilera, A.: Self-organized criticality induced by diversity, Phys. Rev. Lett., 78(8), 1492-1495, 1997.

De Sousa Vieira, M.: Chaos and synchronized chaos in an EQ model, Phys. Rev. Lett., 82, 201-204, 1999.

Dickinson, J., Langford, S., Jensen, L., McVay, G., Kelso, J., and Pantano, C.: Fractoemission from fused silica sodium silicate glasses, J. Vac. Sci. Technol. A, 6, 1084-1089, 1988.

Drossel, B.: Self-organized criticality and synchronization in a forest-fire model, Phys. Rev. Lett., 76, 936-939, 1996.

Ebeling, W., Steuer, R., and Tichener, M.: Partition-based entropies of deterministic and stochastic maps, Stochastic and Dynamics, 1, 1-17, 2001.

Eftaxias, K., Frangos, P., Kapiris, P., Polygiannakis, J., Kopanas, J., Peratzakis, A., Skountzos, P., and Jaggard, D.: Review and a model of pre-seismic electromagnetic emissions in terms of frac- 
tal electrodynamics, Fractals, 12, 243-273, 2004.

Eftaxias, K., Kapiris, P., Dologlou, E., Kopanas, J., Bogris, N., Antonopoulos, G., Peratzakis, A., and Hadjicontis, V.: EM anomalies before the Kozani EQ: A study of their behavior through laboratory experiments, Geophys. Res. Lett., 29(8), 69/1-69/4, 2002.

Eftaxias, K., Kapiris, P., Polygiannakis, J., Bogris, N., Kopanas, J., Antonopoulos, G., Peratzakis, A., and Hadjicontis, V.: Signatures of pending EQ from electromagnetic anomalies, Geophys. Res. Lett., 28, 3321-3324, 2001.

Eftaxias, K., Kapiris, P., Polygiannakis, J., Peratzakis, A., Kopanas, J., and Antonopoulos, G.: Experience of short term EQ precursors with VLF-VHF electromagnetic emissions, Nat. Hazards Earth Syst. Sci., 3, 217-228, 2003.

Eftaxias, K., Kopanas, J., Bogris, N., Kapiris, P., Antonopoulos, G., and Varotsos, P.: Detection of electromagnetic EQ precursory signals in Greece, Proc. Japan Acad., 76(B), 45-50, 2000.

Fujinawa, Y., Takahashi, K., Matsumoto, T., and Kawakami, N.: Experiments to Locate Sources of Earthquake-related VLF Electromagnetic Signals, Proc. Japan Acad., 73(B), 33-38, 1997.

Garcimartin, A., Guarino, A., Bellon, L., and Ciliberto, S.: Statistical analysis of fracture precursors, Phys. Rev. Lett., 79, 79-86, 1997.

Gil, L. and Sornette, D.: Landau-Ginzburg theory of self-organized criticality, Phys. Rev. Lett., 76, 3991-3994, 1996.

Gilden, D., Thornton, T., and Mallon, M.: 1/f noise in human cognition, Science, 267, 1837-1839, 1995.

Gluzman, S. and Sornette, D.: Log-periodic route to fractal functions, Phys. Rev. E, 65, 36142-1-26142-19, 2001.

Golomb, D. and Rinzel, D.: Dynamics of globaly coupled inhibitory neurons with heterogeneity, Phys. Rev. E, 48, 48104814, 1993.

Gong, P., Nikolaev, A., and Van Leeuwen, C.: Scale-invariant fluctuations of the dynamical synchronization in human brain electrical activity, Neuroscience Letters, 336, 33-36, 2003.

Gonzalez, A. and Pantano, C.: Fractoemission during crack propagation in glass, Appl. Phys. Lett., 57, 246-248, 1990.

Grassberger, P. and Procaccia, I.: Characterization of strange attractors, Phys. Rev. Lett., 50, 346-349, 1983.

Grasso, J.-R. and Sornette, D.: Testing self-organized criticality by induced seismicity, J. Geophys. Res., 103, 29 965-29 987, 1998.

Hainzl, S., Zoller, G., and Kurths, J.: Seismic quiescence as an indicator for large EQs in a system of self-organized criticality, Geophys. Res. Lett., 27, 597-600, 2000.

Hausdorff, J. and Peng, C.: Multiscaled randomness: a possible source of 1/f noise in biology, Phys. Rev. E., 54, 2154-2157, 1996.

Heimpel, M.: Critical behavior and the evolution of fault strength during EQ cycles, Nature, 388, 865-868, 1997.

Heneghan C. and McDarby, G.: Establishing the relation between detrended fluctuation analysis and power spectral density analysis for stochastic processes, Phys. Rev. E, 62, 6103-6110, 2000.

Herz, A. and Hopfield, J.: Earthquake cycles and neural reverberations: collective oscillations in systems with pulse-coupled threshold elements, Phys. Rev. Lett., 75, 1222-1225, 1995.

Hirata, T., Satoh, T., and Ito, K.: Fractal structure of spatial distribution of micro fracturing in rocks, Geophys. J. R. Astr. Soc., 90, 369-374, 1987.

Hohemberg, P. and Halperin, B.: Theory of dynamic critical phe- nomena, Rev. Mod. Phys., 49, 435-479, 1977.

Hopfield, J.: Neurons, dynamics and computation, Physics Today, 40, 40-46, 1994.

Huang, Y., Saleur, H., Sammis, C., and Sornette, D.: Precursors, aftershocks, criticality and self-organized criticality, Europhys. Lett., 41, 43-48, 1998.

Iasemidis, L. and Sackellares, J.: Chaos theory and epilepsy, The Neuroscientist, 2(2), 118-125, 1996.

Iasemidis, L.: Epileptic seizure prediction and control, IEEE Transactions on Biomedical Engineering, 50, 548-558, 2003.

Ivanov, P., Amaral, L., Goldberger, A., Havlin, S., Rosenblum, M., Struzik, Z., and Stanley, H.: Multifractality in human heartbeat dynamics, Nature, 399, 461-465, 1999.

Jaeger, H., Nagel, S., and Behringer, R. P.: Granular solids, liquids and gases, Rev. Mod. Phys., 68, 1259-1273, 1996.

Johansen, A. and Sornette, D.: Critical ruptures, Eur. Phys. J. B., 18, 163-181, 2000.

Kadanoff, L., Wolfgang, G., Hamblen, D., Hecht, R., Lewis, E., Palciauskas, V., Rayl, M., and Swift, J.: Static phenomena near critical points: Theory and experiment, Rev. Mod. Phys., 39, 395431, 1967.

Kaiser, G.: A Friendly Guide to Wavelets, Birkhauser, 1994.

Kapiris, P., Balasis, G., Kopanas, J., Antonopoulos, G., Peratzakis, A., and Eftaxias, K.: Scaling similarities of multiple fracturing of solid materials, Nonlin. Processes Geophys., 11, 137-151, 2004.

Kapiris, P., Eftaxias, K., and Chelidze, T.: Electromagnetic signature of prefracture criticality in heterogeneous media, Phys. Rev. Lett., 92(6), 065702/1-4, 2004.

Kapiris, P., Polygiannakis, J., Li, X., Yao, X., and Eftaxias, K.: Similarities in precursory features in seismic shocks and epileptic seizures, Europhys. Lett., 69, 657-663, 2005a.

Kapiris, P., Nomicos, K., Antonopoulos, G., Polygiannakis, J., Karamanos, K., Kopanas, J., Zissos, A., Peratzakis, A., and Eftaxias, K.: Distinguished seismological and electromagnetic features of the impending global failure: Did the 7/9/1999 M5.9 Athens EQ come with a warning?, Earth, Planets and Space, 57, 215-230, 2005b.

Karamanos, K., Peratzakis, A., Kapiris, P., Nikolopoulos, S., Kopanas, J., and Eftaxias, K.: Extracting preseismic electromagnetic signatures in terms of symbolic dynamics, Nonlin. Processes Geophys., 12, 835-848, 2005.

Kolesnikov, J. and Chelidze, T.: The anisotropic correlation in percolation theory, J. Phys. A Math. Gen., 18, 273-275, 1985.

Lai, Y., Osorio, I., Harrison, M., and Frei M.: Correlationdimension and autocorrelation fluctuations in epileptic seizure dynamics, Phys. Rev. E, 65, 031921, 2002.

Lamaingnere, L., Carmona, F., and Sornette, D.: Experimental realization of critical thermal fuses rupture, Phys. Rev. Lett., 77, 2738-2741, 1996.

Langford, S., Dickinson, J., and Jensen, L.: Simultaneous measurements of the electron and photon emission accompanying fracture of single-crystal MgO, J. Appl. Phys., 62, 1437-1449, 1987.

Lei, X., Nishizawa, O., Kusunose, K., Cho, A., Satoh, T., and Nishizawa, O.: Compressive failure of mudstone samples containing quartz veins using rapid ae monitoring: the role of asperities, Tectonophysics, 328, 329-340, 2000.

Lei, X., Masuda, K., Nishizawa, O., Jouniaux, L., Liu, L., Ma, W., Satoh, T., and Kusunose, K.: Detailed analysis of acoustic emission activity during catastrophic fracture of faults in rock, Journal 
of Structural Geology, 26, 247-258, 2004.

Lehnertz, K. and Elger, C.: Can epileptic seizures be predicted? Evidence from nolinear time series analysis of brain electrical activity, Phys. Rev. Lett., 80(22), 5019-5022, 1998.

Lehnertz, K. and Elger, C.: Spatio-temporal dynamics of the primary epileptogenic area in temporal lobe epilepsy characterized by neuronal complexity loss, Electroenceph. Neurophysiol., 95, 108-117, 1995

Lempel, A. and Ziv, J.: On the complexity of finite sequences, IEEE Trans. Inform. Theory, 22, 75-81, 1976.

Leung, K., Andersen, J., and Sornette, D.: Self-organized criticality in stick-slip models with periodic boundaries, Phys. Rev. Lett., 80(9), 1916-1919, 1998.

Linkenkaer-Hansen, K., Nikouline, V., Palva, J., and Ilmoniemi, R.: Long-range temporal correlations and scaling behavior in human brain oscillations, Journal of Neuroscience, 21(4), 1370-1377, 2001.

Litt, B. and Javier, E.: Prediction of epileptic seizures, The Lancet Neurology, 1, 22-30, 2002.

Lomnitz, C.: Fundamentals of Earthquake Predictions, Willey, Ashurst, UK, 1994.

Lux, T. and Marchesi, M.: Scaling and criticality in a stochastic multi-agent model of a financial market, Nature, 397, 498-500, 1999.

Main, I. and Al-Kindy, F.: Entropy, energy and proximity to criticality in global EQ populations, Geophys. Res. Lett., 29, 25/1-25/4, 2002.

Malamud, B., Morein, G., and Turcotte, D.: Forest fires: an example of self-organized critical behavior, Science, 281, 1840-1842, 1998.

Mandelbrot, B.: The fractal geometry of nature, Freeman, New York, 1982.

Mantegna, R. and Stanley, H.: Scaling behavior in the dynamics of an economic index, Nature, 376, 46-49, 1995.

Marodi, M., d' Ovidio, F., and Vicsek, T.: Synchronization of oscillators with long range interaction: phase transition and anomalous finite size effects, Phys. Rev. E, 66, 011109-1-011109-8, 2002.

Maslov, S., Paczuski, M., and Bak, P.: Avalanches and 1/f noise in evolution and growth models, Phys. Rev. Lett., 73, 2162-2165, 1994.

Mavromatou, C., Hadjicontis, V., Ninos, D., Mastrogiannis, D., Hadjicontis, E., and Eftaxias, K.: Understanding the fracture phenomena in inhomogeneous rock samples and ionic crystals, by monitoring the electromagnetic emission during their deformation, Physics and Chemistry of the Earth, 29, 353-357, 2004.

Miura, T. and Nakayama, K.: Spectral analysis of photons emitted during scratching of an insulator surface by a diamond in air, J. Appl. Physics, 88, 5444-5447, 2000.

Mormann, F., Andrzejak, R., Kreuz, T., Rieke, C., David, P., Elger, C., and Lehnertz, K.: Automated detection of a pre-seizure state based on a decrease in synchronization in intracranial electroencephalogram recordings from epilepsy patients, Phys. Rev. E, 67, 021912.1-021912.9, 2003.

Mousseau, N.: Synchronization by disorder in coupled systems, Phys. Rev. Lett., 77, 968-971, 1996.

Newman, W. and Turcotte, D.: A simple model for the EQ cycle combining self-organized complexity with critical point behavior, Nonlin. Processes Geophys., 9, 453-461, 2002.
Nicolis, J.: Chaos and Information Processing, World Scientific, Singapore, 1991.

Nikolopoulos, S., Kapiris, P., Karamanos K., and Eftaxias, K.: A unified approach of catastrophic events, Nat. Hazards Earth Syst. Sci., 4, 615-637, 2004.

Peng, C., Havlin, S., Stanley, H., and Goldberger, A.: Quantification of scaling exponents and crossover phenomena in nonstationary heartbeat timeseries, Chaos, 5, 82-87, 1995.

Plenz, D.: When inhibition goes incognito: feedback interaction between spiny projection neurons in striatal function, TRENDS in Neurosciences, 26(8), 436-443, 2003.

Ponomarev, A., Zavyalov, A., Smirnov, V., and Lockner, D.: Physical modelling of the formation and evolution of seismically active fault zones, Tectonophysics, 277, 57-81, 1997.

Pruessner, G. and Jensen, H.: Anisotropy and universality: The Oslo model; the ricepile experiment, and the quenched EdwardsWilkinson equation, Phys. Rev. Lett., 91, 244303-1-244303-4, 2003.

Rabinovitch, A., Frid, V., and Bahat, D.: Gutenberg-Richter-type relation for laboratory fracture-induced electromagnetic radiation, Phys. Rev. E, 65, 11401/1-11401/4, 2001.

Rundle, J., Klein, W., Gross, S., and Turcotte, D.: Boltzmann fluctuations in numerical simulations of nonequilibrium lattice threshold systems, Phys. Rev. Lett., 75, 1658-1661,1995.

Rundle, J., Tiampo, K., Klein, W., and Sa Martins, J.: Selforganization in leaky threshold systems: the influence of nearmean field dynamics and its implications for EQs, neurology, and forecasting, PNAS, 99, 2514-2521, 2002.

Sackellares, J., Iasemidis, L., Shiau, D., Gilmore, R., and Roper, S.: Epilepsy - when chaos fails, in: Chaos in the Brain, pages 112-133, edited by: Lechnertz, K., Ambhold, J., Grassberger, P., and Elger, C. E., World Scientific, Singapore, 2000.

Sahimi, M. and Arbadi, S.: Scaling laws for fracture of heterogeneous materials and rock, Phys. Rev. Lett., 77, 3689-3692, 1996a.

Sahimi, M. and Arbadi, S.: Scaling laws for fracture of heterogeneous materials and rock, Phys. Rev. Lett., 77, 3689-3692, 1996b.

Sammis, C. and Sornette, D.: Positive feedback, memory, and the predictability of EQs, PNAS, 99, 2501-2508, 2002.

Sammis, C., Sornette, D., and Saleur, H.: Complexity and EQ forecasting, in Reduction and Predictability of Natural Disasters, SFI studies: in: the Sciences of complexity, volume XXV, pages 143-156, edited by: Rundle, J., Klein, W., and Turcotte, D., Addison-Wesley, Reading, Mass., 1996.

Sharon, E. and Fineberg, J.: Confirming the continuum theory of dynamic brittle fracture for fast cracks, Nature, 397, 333-335, 1999.

Sharon, E. and Fineberg, J.: Microbranching instability and the dynamic fracture of brittle material, Phys. Rev. B, 54(10), 71287139, 1996.

Sornette, D. and Andersen J.: Scaling with respect to disordered in time-to-failure, Eur. Phys. J. B., 1, 353-357, 1998.

Sornette, D. and Sammis, C.: Complex critical exponents from renormalization group theory of EQs: Implications for EQ predictions, J. Phys. I., 5, 607-619, 1995.

Sornette, D., Johansen, A., and Bouchaud, J.-P.: Stock market crashes, precursors and replicas, J. Phys. I F France, 6, 167-175, 1996. 
Sornette, D.: Critical Phenomena in Natural Sciences, Springer, 2000.

Sornette, D.: Predictability of catastrophic events: Material rupture, EQs, turbulence, financial crashes, and human birth, PNAS, 99, 2522-2529, 2002.

Sornette, D. and Vanneste, C.: Dynamics and memory effects in rupture of thermal fuse networks, Phys. Rev. Lett., 68, 612-615, 1992.

Stanley, H.: Exotic statistical physics: Applications to biology, medicine, and economics, Physica A, 285, 1-17, 2000.

Stanley, H.: Scaling, universality, and renormalization: Three pillars of modern critical phenomena, Reviews of Modern Physics, 71(2), S358-S366, 1999.

Takens, F.: Detecting strange attractors in turbulence, in: Dynamical systems and turbulence, Warwick 1980: Proceedings of symposium held at the University of Warwick 1979-1980, 366-381, Springer-Verlag, Berlin, 1981.

Takeuchi, A. and Nagahama, H.: Scaling laws between seismoelectric/magnetic fields and EQ magnitude, Terra Nova, 16, 152156, 2004.

Tichener, M.: Deterministic computation of string complexity, information and entropy, in: Intern. Symp. on Information Theory, MIT, Boston, August 16-21, 1998.
Tichener, M.: A measure of information, in: IEEE Data Compression Conference, Snowbird, March 2000.

Tsukamoto, N., Miyazaki, S., and Fujisaka, H.: Synchronization and intermittency in three-coupled chaotic oscillators, Phys. Rev. E, 67, 16212-1-16212-15, 2003.

Turcotte, D.: Fractals and chaos in geology and geophysics, Cambridge University Press, 1992

Usher, M., Stemmler, M., and Olami, Z.: Dynamic pattern formation leads to $1 / f$ noise in neural populations, Phys. Rev. Lett., 74, 326-329, 1995.

Vicsek, T.: A question of scale, Nature, 411, 421, 2001.

Vicsek, T.: The bigger picture, Nature, 418, 131, 2002.

Worrell, G., Cranstoun, S., Litt, B., and Echauz, J.: Evidence for self-organized criticality in human epileptic hippocampus, Neurophysiology, Basic and Clinical, 13, 1-6, 2002.

Wu, L., Wu, Y., Liu, S., Li, G., and Li, Y.: Infrared radiation of rock impacted at low velocity, Rock Mechanics and Mining Sciences, 41, 321-327, 2004.

Yang, X., Du, S., and Ma, J.: Do EQs exhibit self-organized criticality: Phys. Rev. Lett., 92, 228501-1-2228501-4, 2004.

Zhao, X. and Chen, T.: Type of self-organized criticality model based on neural networks, Phys. Rev. E, 65, 026114-1-026114-6, 2002 . 